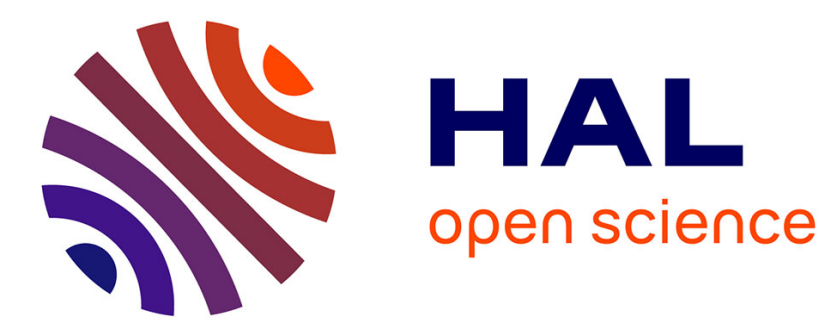

\title{
Le principe de précaution comme norme de l'action publique ou la proportionnalité en question \\ Olivier Godard
}

\section{To cite this version:}

Olivier Godard. Le principe de précaution comme norme de l'action publique ou la proportionnalité en question. 2003. hal-00242985

\section{HAL Id: hal-00242985 \\ https://hal.science/hal-00242985}

Preprint submitted on 6 Feb 2008

HAL is a multi-disciplinary open access archive for the deposit and dissemination of scientific research documents, whether they are published or not. The documents may come from teaching and research institutions in France or abroad, or from public or private research centers.
L'archive ouverte pluridisciplinaire HAL, est destinée au dépôt et à la diffusion de documents scientifiques de niveau recherche, publiés ou non, émanant des établissements d'enseignement et de recherche français ou étrangers, des laboratoires publics ou privés. 


\section{ECOLE POLYTECHNIQUE}

CENTRE NATIONAL DE LA RECHERCHE SCIENTIFIQUE

Le principe de précaution comme norme de l'action publique,ou la proportionnalité en question

Olivier Godard

Novembre 2003

Cahier $^{\circ}{ }^{2} 2003-025$

\section{LABORATOIRE D'ECONOMETRIE}

1rue Descartes F-75005 Paris

(33) 155558215

http://ceco.polytechnique.fr/

mailto:labecox@poly.polytechnique.fr 


\title{
Le principe de précaution comme norme de l'action publique,ou la proportionnalité en question
}

\author{
Olivier Godard ${ }^{1}$
}

Novembre 2003

Cahier $n^{\circ}$ 2003-025

Résumé: L'introduction du principe de précaution en France a donné naissance à deux conceptions antagonistes. La première, correspondant à la définition légale et à la doctrine européenne, promeut une prévention précoce mais proportionnée de risques potentiels. La seconde, portée par des ONG et relayée par l'usage courant en situation de crise, demande la preuve de l'innocuité et vise asymptotiquement l'éradication des risques par l'accumulation de mesures précautionneuses. Cette situation a entretenu une confusion entre deux idées : agir de façon précoce, sans attendre des certitudes scientifiques, et agir de façon plus précautionneuse. L'article dresse un tableau des conceptions en présence puis montre en quoi la " règle d'abstention " n'est pas défendable, même lorsqu'on atténue ses exigences. Il montre ensuite comment une mauvaise structuration du problème de décision crée un artefact par lequel la précocité dans le temps scientifique induit mécaniquement une inflation des risques via l'aggravation des dommages potentiels perçus. Pour s'en prémunir, il faut s'en tenir à deux garde-fous : instituer la prise en compte des avantages potentiels au même titre que les dommages potentiels ; proportionner les actions de prévention à la plausibilité scientifique des hypothèses de risque de façon à donner de moindres effets pratiques aux conjectures non étayées qu'aux hypothèses confortées par un faisceau d'éléments scientifiques.

\begin{abstract}
:
In France the precautionary principle gave birth to two basic opposite interpretations. The first one, fixed in the French legal framework and developed by the European doctrine, supports an early but proportionate consideration of potential hazards. The second one has been backed by environmental NGOs and disseminated into current use by media and political circles under crisis circumstances. It asks for a proof of no-risk and aims at eradicating all sources of hazards to the possible extent. As a result, a basic confusion arose between two ideas: to take early preventive action, and to accumulate more stringent preventive measures. Firstly, the paper sketches the landscape of competing concepts. It then shows why the "abstention rule" cannot be supported, even when its requirements are attenuated. It also identifies how a wrong framing of the decision problem leads a specific artefact to arise, by which earliness in the scientific time induces an overall inflation of risks through a perception of increased potential damage. To keep the precautionary principle on the right track, two requirements are to be met: enforcing the consideration of potential benefits the same way as potential hazards; proportioning preventive measures to the scientific plausibility of hypotheses of risks, in order to give a lower weight to assumptions deprived of significant evidence.
\end{abstract}

Mots clés : $\quad$ Incertitude, risques, décision, principe de précaution, évaluation

Key Words : Uncertainty, hazards, decision, precautionary principle, assessment

Classification JEL: A13, D81, I18, K32

\footnotetext{
${ }^{1}$ Laboratoire d'Econométrie, CNRS et Ecole polytechnique
} 


\section{INTRODUCTION}

En une dizaine d'années le principe de précaution (désigné dans la suite de l'article comme le $P P$ ) est devenu une norme de référence pour les actions et politiques publiques dans le domaine de la protection de l'environnement puis, par extension, de la sécurité alimentaire et de la santé publique. Ce principe vise l'attitude à adopter face à des « risques collectifs potentiels ». L'expression « risques $^{1}$ potentiels » désigne à la fois des dangers dont l'existence même, au moment où ils sont appréhendés, n'est pas scientifiquement établie, n'étant ni prouvée ni réfutée, et ceux pour lesquels, en dépit d'une existence établie, il n'est pas encore possible d'établir de façon scientifique, par l'analyse logique ou par l'observation statistique d'événements répétés, une distribution de probabilité d'occurrence ${ }^{2}$. L'expression risques collectifs désigne des menaces d'atteintes qui affectent des biens collectifs (environnement) ou qui concernent de larges groupes de personnes du fait du comportement d'autres agents ou de phénomènes naturels. Le champ ainsi délimité se distingue de celui des risques privés comme de celui qui a été dessiné pour les actions et politiques de prévention de risques collectifs scientifiquement établis (risques avérés). Pour ces derniers l'action publique et individuelle de prévention s'appuie sur la connaissance en probabilité de l'exposition d'une population au danger. Le même fond de risques connus, à probabilité définie, forme le socle de l'économie de l'assurance, sans que cette dernière soit totalement empêchée d'aller audelà ${ }^{3}$. La distinction entre politiques de précaution et politiques de prévention et, par effet de retour, sur les attributs des principes respectifs de précaution et de prévention, se fait donc en premier lieu à partir des modalités de l'action visant des risques collectifs, c'est-à-dire du tableau scientifique disponible et des ressources cognitives mobilisables dans chaque type de

\footnotetext{
${ }^{1}$ L'emploi du mot 'risque' suscite parfois des querelles terminologiques. Si on définit le risque comme «une fonction de la probabilité et de la gravité d'un effet néfaste du fait de la présence d'un danger », comme le fait le règlement européen 78/2002 sur la législation alimentaire et la sécurité des denrées alimentaires (2002), l'expression 'risques potentiels' n'a pas de consistance puisqu'elle désigne des situations pour lesquelles il n'est pas possible d'énoncer une probabilité objectivement et scientifiquement établie. Si on définit le risque par le caractère aléatoire des conséquences d'une action, l'aléa pouvant se manifester de façon symétrique autour d'une valeur centrale, l'expression 'risques potentiels' est également impropre car elle se rapporte exclusivement à des dangers, c'est-à-dire à la possibilité de dommages. En fait l'usage et le débat social ont imposé un sens du mot risque distinct de celui de la théorie de la décision: c'est le danger possible, la menace de péril ou de catastrophe. C'est ainsi que s'est développé depuis vingt-cinq ans un champ d'études du 'risque technologique majeur' qui n'entretient pas de rapport définitionnel avec la mise en œuvre du calcul de probabilités (Lagadec, 1981). Et quand la sociologie s'approprie la thématique du risque, elle en redéfinit le concept pour en faire la clé de voûte de nouveaux rapports sociaux en rupture avec les fondements de la société industrielle (Beck, 2001). Je suivrai dans ce texte l'usage devenu courant, car c'est celui qui structure le débat sur l'application du principe de précaution.

${ }^{2}$ Cette situation n'empêche évidemment pas un agent quelconque de se donner sa propre distribution de probabilités, qui résultera alors d'un composé d'information et de croyances. Une telle opération peut déclencher une prise de décision personnelle ou déboucher sur la formation d'une opinion. Mais, en tant que telle, elle est dénuée de pertinence directe pour fonder l'action publique envers un risque collectif, la scène publique impliquant une exigence de justification partageable par les autres membres de la société, que cette exigence s'adresse aux citoyens qui font différentes demandes ou au dirigeant qui doit rendre des comptes. Cette exigence de justification est ignorée par les modèles de décision qui renvoient simplement aux croyances et préférences du décideur public. Voilà pourquoi je n'utilise pas un formalisme bayesien pour caractériser le $P P$.

${ }^{3}$ Le milieu de l'assurance s'est cependant aventuré, dans la période récente, dans la couverture de risques jugés a priori non assurables car éloignés d'un contexte probabiliste classique, notamment en recherchant une articulation avec les marchés financiers. Voir la Partie 3 « Assurance » du Traité de Godard, Henry, Lagadec et Michel-Kerjan [2002, pp. 359-548].
} 
contexte.

Du fait de la coexistence, dans le cas le plus général relevant du $P P$, d'une incertitude d'existence et d'une incertitude d'occurrence, le champ d'application du principe de précaution ne se prête pas au calcul objectif des risques ${ }^{4}$. En particulier, des risques qui ne sont que potentiels peuvent se révéler ultérieurement graves ou massifs ; il serait donc erroné d'assimiler risques potentiels et petits risques. L'impossibilité d'adopter une mise en forme probabiliste établie de façon objective et scientifique conduit l'action publique à se guider en fonction d'autres repères : aux procédures d'expertise scientifique s'adjoignent les procédures de débat public et de concertation avec les parties intéressées. Cependant, les actions engagées sous l'égide du principe de précaution n'ont pas pour autant à rompre avec les exigences générales de la raison, même si cette dernière doit se résoudre à recourir à d'autres voies d'atteinte du raisonnable que celles du calcul économique. Cette situation soulève une question délicate : comment comprendre l'exigence générale de proportionnalité, qui est au coeur de l'idée même de raison, dans le contexte de politiques de précaution ? Telle est la principale question que cet article vise à éclairer.

A cette fin, je dresserai d'abord le tableau des conceptions en présence, polarisé autour des deux conceptions antagonistes de base de ce principe (section 1). La première correspond à la définition légale retenue en France par la loi Barnier de renforcement de la protection de l'environnement (1995) et a été développée par la doctrine européenne. Elle s'attache à promouvoir une prévention précoce mais proportionnée de risques potentiels. La seconde, portée par des ONG et relayée par l'usage courant en situation de crise, pousse à la surenchère précautionneuse, vise asymptotiquement l'éradication des risques et peut aller jusqu'à demander la preuve de l'innocuité. Sous cette forme extrême, elle s'incarne alors dans une règle d'abstention devant le risque. La coexistence de ces deux compréhension du $P P$ a entretenu une confusion entre deux idées : agir de façon précoce, sans attendre, et agir de façon plus précautionneuse. L'article montre ensuite en quoi la règle d'abstention n'est pas défendable, même lorsqu'on atténue ses exigences (section 2). Il montre surtout comment une mauvaise structuration du problème de décision crée un artefact par lequel la précocité de la prise en compte du risque induit mécaniquement un accroissement de la gravité perçue de tout risque potentiel, ce qui provoque une inflation des risques perçus (section 3). Pour s'en prémunir, il faut s'en tenir à deux garde-fous : instituer la prise en compte des avantages procurés par les biens et activités supports du risque, au même titre que les dommages potentiels ; proportionner les actions de prévention à la consistance ou plausibilité scientifique des hypothèses de risque de façon à donner de moindres effets pratiques aux conjectures non étayées qu'aux hypothèses confortées par un faisceau d'éléments scientifiques et empiriques.

\section{L'ANTAGONISME DES CONCEPTIONS EN PRÉSENCE}

Le moins que l'on puisse dire est qu'il a régné depuis le début des années 90 une certaine confusion sur le principe de précaution à la fois dans le débat public, le débat d'experts et la prise de parole d'acteurs parties prenantes de la décision publique. En dépit des efforts de

\footnotetext{
${ }^{4}$ Il est certes possible de procéder à des calculs sur des séquences bien connues, ou à l'intérieur de scénarios, mais ces calculs ne peuvent pas déboucher à eux seuls sur la mise en évidence d'une meilleure solution.
} 
clarification théorique et doctrinale de la part des chercheurs ${ }^{5}$, de rapports d'expertise publique (rapport Kourilsky-Viney au Premier ministre, 2000) et des institutions ayant des responsabilités de gestion ${ }^{6}$, le contenu de la nouvelle norme n'est pas encore stabilisé en pratique. Inégalement reconnu par différents ordres juridiques (droit international, droit communautaire, droit interne français), son interprétation est l'objet de tensions et de divergences qui se manifestent à l'occasion de décisions concrètes de gestion des risques ou d'interprétation de la jurisprudence, en particulier lorsqu'il existe des enjeux de commerce international. Par exemple, le bien-fondé des mesures d'interdiction de l'importation de bœuf américain élevé aux hormones de la part de l'Union européenne a pu être contesté, en premier lieu par le gouvernement américain (Noiville, 2000).

\section{Le Conseil d'Etat contre la loi Barnier !}

Le champ du débat est bien balisé par deux définitions qui ont été importantes pour camper le $P P$ en France. La première est celle donnée par la Loi 95-101 de renforcement de la protection de l'environnement, dite Loi Barnier, et inscrite dans le Code de l'environnement (article L 110-1) :

« Le principe de précaution, selon lequel l'absence de certitudes, compte tenu des connaissances scientifiques et techniques du moment, ne doit pas retarder l'adoption de mesures effectives et proportionnées visant à prévenir un risque de dommages graves et irréversibles à l'environnement à un coût économiquement acceptable $\gg^{7}$.

La seconde est celle que s'étaient donnée les rapporteurs du Conseil d'État dans leur rapport public 1998 consacré au droit de la santé :

«Ce nouveau concept se définit par l'obligation pesant sur le décideur public ou privé de s'astreindre à une action ou de s'y refuser en fonction du risque possible. Dans ce sens, il ne lui suffit pas de conformer sa conduite à la prise en compte des risques connus. Il doit, en outre, apporter la preuve, compte tenu de l'état actuel de la science, de l'absence de risque ».

(Conseil d'État, 1998, p. 256).

Apporter la preuve de l'absence de risque! On retrouve ici une formulation caractéristique d'une règle d'abstention, forme extrême d'une approche précautionneuse visant à s'approcher asymptotiquement de l'éradication des risques. Godard [1997, 2002a] et Godard, Henry, Lagadec et Michel-Kerjan [2002] ont montré la différence conceptuelle

\footnotetext{
${ }^{5}$ Voir en particulier les ouvrages coordonnés par O’Riordan \& Cameron [1994], Godard [1997], Raffensperger et Tickner [1999], Zaccaï et Missa [2000] et les ouvrages collectifs de Ewald, Gollier, de Sadeleer [2001] et Godard, Henry, Lagadec et Michel-Kerjan [2002].

${ }^{6}$ Dans le contexte européen, les textes importants sont la Communication de la Commission sur le principe de précaution (février 2000), la Résolution sur le principe de précaution adoptée par le Sommet des chefs d'État et de gouvernement réunis à Nice en décembre 2000 et le règlement 178/2002 sur la sécurité alimentaire.

${ }^{7}$ Le projet de loi constitutionnelle adopté par le gouvernement le 25 juin 2003 en vue d'adosser une Charte de l'environnement à la Constitution française inclut l'énoncé suivant: "Lorsque la réalisation d'un dommage, bien qu'incertaine en l'état des connaissances scientifiques, pourrait affecter de manière grave et irréversible l'environnement, les autorités publiques veillent, par application du principe de précaution, à l'adoption de mesures provisoires et proportionnées afin d'éviter la réalisation du dommage ainsi qu'à la mise en oeuvre de procédures d'évaluation des risques encourus ».
} 
importante entre cette règle d'abstention devant le risque et ce que demande le $P P$ dans les définitions qui lui ont été apportées dans différents textes de droit ou de doctrine, soit internationaux (Déclaration de Rio sur l'environnement et le développement de 1992), soit communautaires (voir l'encadré présentant la résolution du Sommet européen de Nice en décembre 2000), soit interne (Loi Barnier 95-101 ; rapport Kourilsky-Viney). La confusion était donc troublante de la part du Conseil d'État. On comprend mieux cependant ce que fut la démarche des rapporteurs quand on découvre dans la suite du rapport que cette définition décalée, qui reprenait une formulation avancée par des organisations militantes, jouait le rôle d'un « repoussoir»: les rapporteurs recommandaient in fine de ne pas modifier les bases existantes de la responsabilité médicale et de ne pas faire de ce principe de précaution-là un nouveau fondement de cette responsabilité, au côté des deux fondements reconnus qu'étaient la faute et le risque. Sur la base de la définition qu'ils s'étaient donnée, c'était tout à fait raisonnable.

\section{LA RESOLUTION SUR LE PRINCIPE DE PRECAUTION DU CONSEIL EUROPEEN DE NICE (2000) \\ (d'après Godard, Henry, Lagadec, Michel-Kerjan, [2002], p. 122-123)}

1. Le principe de précaution est applicable au domaine de l'environnement et également à la santé humaine, ainsi que dans les domaines zoo et phytosanitaires; il se place dans la perspective du développement durable.

2. Le principe de précaution s'applique aux politiques et actions de la Communauté et de ses États membres ; il concerne l'action des autorités publiques, tant au niveau des institutions communautaires qu'à celui des États membres.

3. Il y a lieu de recourir au principe de précaution dès lors que la possibilité d'effets nocifs sur la santé ou l'environnement est identifiée et qu'une évaluation scientifique préliminaire sur la base des données disponibles ne permet pas de conclure avec certitude sur le niveau de risque.

4. Pour procéder à l'évaluation des risques l'autorité publique doit se doter d'un cadre de recherche approprié, en s'appuyant notamment sur des comités scientifiques et sur les travaux scientifiques pertinents ; l'autorité publique est responsable de l'organisation de l'évaluation du risque, qui doit être conduite de façon pluridisciplinaire, contradictoire, indépendante et transparente.

5. L'évaluation du risque doit faire ressortir les avis minoritaires éventuels.

6. Les mesures de gestion du risque doivent être prises par les autorités publiques responsables sur la base d'une appréciation politique du niveau de protection recherché.

7. Lors du choix des mesures à prendre pour la gestion du risque, tout l'éventail des mesures permettant d'atteindre le niveau de protection recherché doit être envisagé.

8. La société civile doit être associée et une attention particulière doit être accordée à la consultation de toutes les parties intéressées à un stade aussi précoce que possible.

9. Les mesures prises doivent respecter le principe de proportionnalité en tenant compte des risques à court et à long terme et en visant le niveau élevé de protection recherché.

10. Lorsqu'il existe plusieurs possibilités d'atteindre le même niveau de protection de la santé ou de l'environnement, les mesures les moins restrictives pour les échanges doivent être recherchées.

11. Les mesures devraient être cohérentes avec les mesures déjà prises dans des situations similaires ou utilisant des approches similaires, compte tenu des développements scientifiques les plus récents et de l'évolution du niveau de protection recherché.

12. Les décisions prises au titre du principe de précaution doivent être réexaminées en fonction de l'évolution des connaissances scientifiques. À cette fin, le suivi des effets de ces décisions doit être assuré et des recherches complémentaires doivent être menées pour réduire le niveau d'incertitude.

Néanmoins, aux yeux de lecteurs non avertis, se trouvait consacrée une confusion fâcheuse entre les deux concepts, même si, à la différence de la définition légale de la Loi 
Barnier, les réflexions du Conseil d'État n'ont pas de portée juridique directe. Par ailleurs, force est de reconnaître que l'assimilation du principe de précaution à une règle d'abstention correspond aux demandes pressantes d'ONG de protection de l'environnement, comme Greenpeace, ou de victimes, lorsque des dommages à la santé humaine se produisent. La tension entre les deux concepts aimante donc le champ du débat intellectuel et social sur le $P P$ et constitue un bon point de départ pour l'analyse.

\section{Une clarification graphique}

Afin de marquer avec netteté en quoi les deux conceptions principales considérées diffèrent, examinons la représentation graphique suivante (figure 1). Sur l'ordonnée est représenté le moment dans le temps scientifique où les autorités responsables décident de prendre en compte un risque potentiel identifié en vue d'adopter une politique de prévention. La graduation de cet axe du temps est celle, non du temps historique, mais du temps de maturation de la connaissance scientifique.

En position haute, éloignée de l'origine, se trouve le moment où un risque est scientifiquement avéré dans son existence et dans sa probabilité d'occurrence. Cette position correspond au concept de risque retenu par Frank Knight [1921] dans son opposition à celui d'incertitude. Si les autorités décident de ne considérer que les risques qui sont parvenus à ce stade de maturité des connaissances, cela signifie qu'elles délaissent les risques qui ne bénéficient pas de la même maturité scientifique. Elles ne quittent pas le domaine et la temporalité classiques qui sont ceux de la prévention strictement définie.

En descendant le long de cet axe des ordonnées, sont repérés les moments de prise en compte de risques qui se trouvent à un stade de moins en moins consistant du point de vue de la connaissance scientifique: d'abord celui de la forte présomption assise sur un faisceau convergent d'éléments théoriques et empiriques, puis un peu plus bas celle de l'hypothèse jugée plausible par une majorité de scientifiques sans disposer d'un étayage empirique, puis encore plus bas, celle de la simple hypothèse qui, certes compatible avec l'état des connaissances, n'a été ni écartée ni établie, mais seulement formulée. Près de l'origine, on approche l'état d'ignorance concernant l'existence de risques potentiels qui ne sont même pas identifiés : en l'état actuel des connaissances et des tests disponibles, rien ne peut être affirmé quant à l'existence de tels risques, sans pouvoir écarter qu'un danger inconnu puisse exister (voir l'encadré sur la graduation qualitative de la plausibilité scientifique).

Sur l'axe des abscisses, on range les différents types de mesures qui peuvent être prises au nom du $P P$, allant du plus sévère, l'interdiction définitive, au moins sévère pour les libertés individuelles et en particulier la liberté d'entreprise, qui est l'organisation d'une veille. Les grades intermédiaires sont: la suspension provisoire d'une autorisation, l'imposition d'une procédure d'autorisation préalable à la mise sur le marché (AMM), différentes restrictions sur les usages autorisés, les conditions et l'intensité de l'usage (par exemple, fixation de valeurs limites), des actions incitatives de type économique (taxes, permis négociables), des actions de signalisation (labels, étiquetage) et d'information du grand public ou seulement de publics spécialisés (groupes-cibles, médecins du travail, etc.) et finalement le lancement de programmes de recherche ad hoc. 

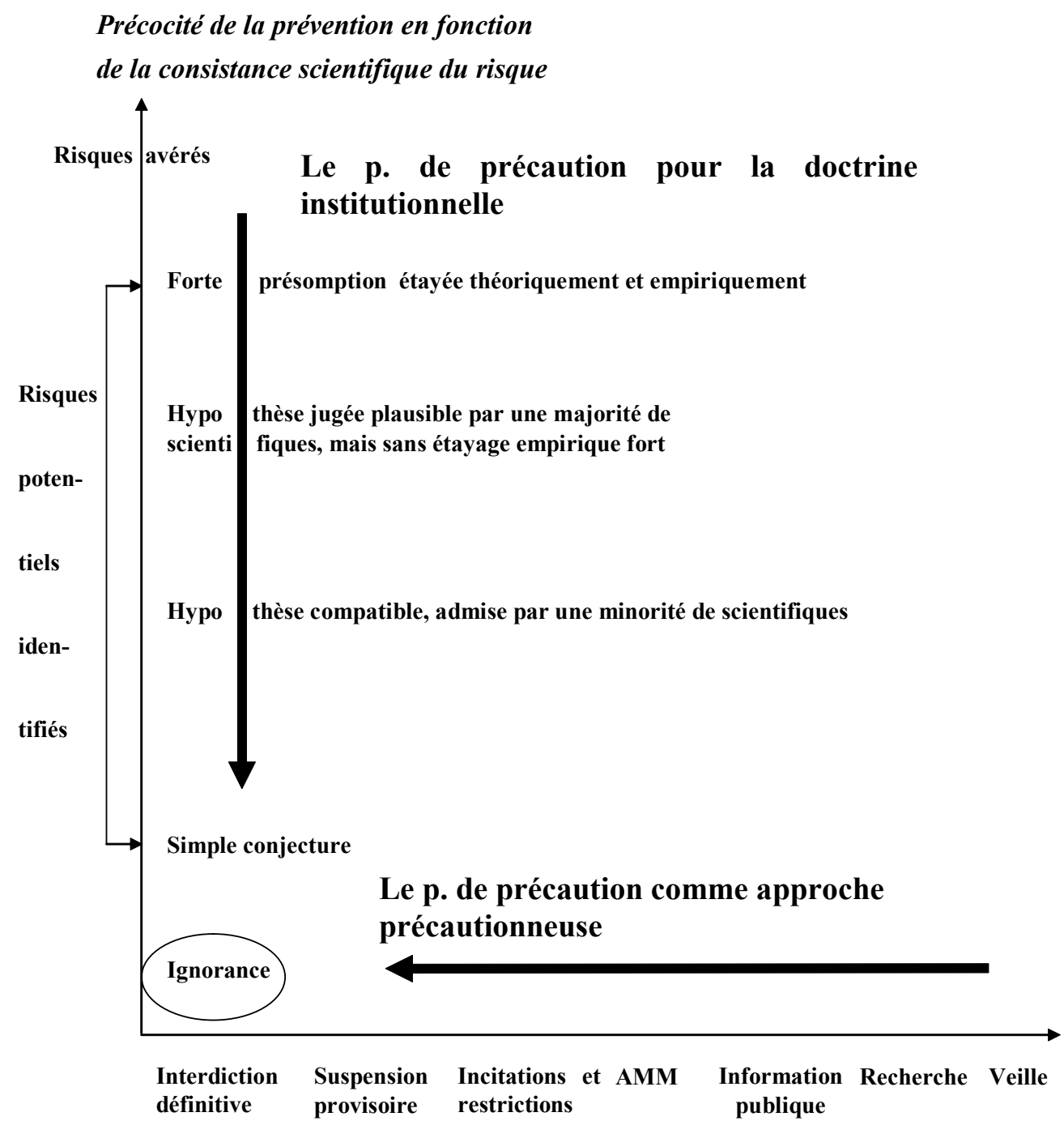

(+) Sévérité décroissante des mesures de précaution

(-)

Figure 1 : Les deux conceptions du principe de précaution

Incidemment, cette simple liste suggère que le $P P$ ne relève pas d'une logique binaire à laquelle il est parfois faussement réduit. Lorsque la question principale aux yeux d'interlocuteurs semble être "faut-il appliquer ou ne pas appliquer le $P P$ ? », cela signifie généralement pour eux : faut-il interdire ou ne pas interdire, comme s'il s'agissait de la seule action envisageable?

Comment situer conceptuellement le $P P$ sur la figure 1 ? Il est manifeste que les deux conceptions de base s'y distinguent par l'idée principale qu'elles véhiculent. Le $P P$ de la doctrine institutionnelle $P P_{\text {inst }}$, dont l'idée principale est «l'absence de certitude ne doit pas retarder l'adoption de mesures...», se traduit par un déplacement vertical vers l'origine du moment, dans le temps scientifique, où le risque est pris en compte par les gestionnaires. Dans la mesure où il ne peut prendre en compte que des risques identifiés, le champ est borné inférieurement par le grade «simple conjecture », sans plonger jusqu'à «l'ignorance». À 
l'opposé, le $P P$ propre à l'approche « précautionneuse » $P P_{\text {prec}}$, qui tend vers une conception éradicatrice $P P_{\text {eradic }}$ du risque, voire la règle d'abstention $P P_{a b s t}$, et dont l'idée principale est « la précaution, c'est adopter des mesures plus sévères de prévention », se traduit par un déplacement horizontal vers l'origine à partir d'un point représentant les mesures qui auraient été adoptées dans le cadre d'une politique classique de prévention.

\section{DES GRADUATIONS DE LA CONSISTANCE SCIENTIFIQUE DES HYPOTHESES DE RISQUES}

Le rapport Kourilsky-Viney [2000] propose de définir deux seuils critiques de plausibilité scientifique, chaque franchissement de seuil ayant des conséquences importantes pour le domaine admissible d'actions de prévention à considérer :

(1) Les risques hypothétiques correspondent à une plausibilité faible. Il s'agit d'hypothèses de travail qui ne sont encore étayées par aucune donnée factuelle. Ils ne sont justiciables que d'une recherche de base et d'une veille.

(2) Les risques potentiels plausibles ont une plausibilité moyenne. Faits et observations disponibles débouchent sur des hypothèses jugées recevables par une partie non négligeable de la communauté scientifique, tout en ayant besoin d'être confirmées ou démenties par des observations de terrain ou des données expérimentales. Ils appellent le lancement d'actions de recherche ciblées afin de lever les points d'incertitude, mais ne justifient pas encore des mesures restrictives des libertés.

(3) Les risques potentiels étayés ont une forte plausibilité. Encore non prouvée expérimentalement, leur existence apparaît très plausible au vu d'un faisceau convergent d'observations de terrain, de travaux de modélisation et d'expériences in vitro. Une grande majorité de la communauté scientifique partage la même conviction quant à l'existence de ces risques. Seul ce dernier type de risques pourrait, aux yeux des auteurs, justifier l'engagement de mesures de prévention restrictives des libertés.

Cette typologie donne un grand poids décisionnel aux éléments empiriques d'étayage, ce qui est peu convaincant pour la classe des risques à effets fortement retardés mais faiblement réversibles, comme le changement climatique planétaire. La grille doit être encore enrichie et le curseur de l'action peut légitimement être déplacé. A cet égard, Bernard Chevassus-Au-Louis [2000] propose de distinguer deux dimensions supplémentaires de la plausibilité : la quantité d'information mobilisable autour d'une hypothèse de risque (nombre de travaux indépendants) et le degré de consensus de la communauté scientifique à son sujet.

De leur côté, Godard et al. [2002] proposent la graduation suivante pour qualifier la maturité scientifique des connaissances des risques :

- la simple conjecture scientifique,

- l'hypothèse non étayée, non réfutée et compatible avec l'état des savoirs scientifiques,

- l'hypothèse non étayée mais résultant d'une méthode scientifique acceptée,

- l'hypothèse étayée par des travaux scientifiques de modélisation et/ ou simulation, des observations empiriques ou des résultats expérimentaux ayant la validité d'indices et acceptée ou soutenue par une minorité de scientifiques,

- l'hypothèse scientifique étayée, empiriquement ou par modélisation, et acceptée par une majorité de scientifiques,

- l'hypothèse validée de façon isolée, à confirmer, qu'il s'agisse d'une démonstration expérimentale ou d'une découverte, demandant à être reproduite, confirmée, ou critiquée,

- le résultat majoritairement accepté par la communauté scientifique, mais encore soumis aux critiques ou réserves d'une minorité scientifique,

- le résultat avéré, accepté par la communauté scientifique quant à l'existence du risque. 
La question soulevée par cette opposition est de savoir si le fait qu'une même expression abrite deux concepts aussi différents est simplement le signe des tensions qui traversent l'action publique au sein de ce que Ulrich Beck [2001] a appelé des sociétés du risque, ou s'il existe une fonction liant les deux concepts l'un à l'autre de telle façon qu'un déplacement vertical dans le temps scientifique doive entraîner un déplacement horizontal sur l'axe des actions. Ce serait le cas si une prise en compte plus précoce des risques devait systématiquement se traduire par des mesures plus sévères de prévention. Cette question sera reprise en section 3.

\section{Conception tactique et conception stratégique du principe de précaution}

Au-delà de l'opposition de base qui vient d'être explicitée, on peut discerner dans des textes de doctrine et ceux qui ont une valeur d'engagement politique une seconde opposition entre ce que j'appelle une "approche tactique», $P P^{t a c}$, du $P P$ et une «approche stratégique », $P P^{\text {stra }}$. L'approche «tactique» vise à " gérer l'attente d'information », pour reprendre une expression de Treich [1997], autant qu'à gérer le risque. Elle s'inscrit dans le court et le moyen terme et s'organise autour de l'idée de mesures provisoires et révisables en fonction de l'amélioration de la connaissance du risque. Les mesures adoptées doivent donc être réversibles. Cette approche se caractérise par une grande attention accordée à la construction des savoirs scientifiques et à l'évolution des connaissances permise par les programmes de recherche. Compte tenu de la première distinction opérée, cette approche tactique peut prendre des formes différentes selon la conception de base du $P P$ retenue : il s'agira ainsi du moratoire pour le $P P_{a b s}{ }^{t a c}$ mais, pour le $P P_{\text {inst }}{ }^{t a c}$, de mesures préventives n'excluant pas toute prise de risque.

Les approches $P P_{\text {eradic }}{ }^{t a c}$ et $P P_{a b s}{ }^{t a c}$ qui visent l'élimination des sources de risques potentiels avant même que les risques en jeu soient avérés ont une contrepartie : elles obligent à renoncer à la perspective de découvrir la réalité empirique des risques suspectés; elles obligent également à renoncer aux avantages mal connus ou encore inconnus de technologies condamnées de façon précoce sur simple soupçon. De ce fait, la justification des mesures de prévention prises sous leur égide demeure à jamais en suspens. L'approche $P P_{\text {inst }}{ }^{t a c}$ est celle qui correspond explicitement à la doctrine établie par l'Organe de règlements des conflits dans le cadre de l'accord sur les produits sanitaires et phytosanitaires conclu au sein de l'Organisation mondiale du commerce: un pays n'est fondé à prendre et maintenir des mesures de précaution contre des produits importés que si les éléments scientifiques mis en avant sont confortés dans un délai raisonnable par les résultats de programmes de recherche scientifique qu'il aura lancés (Noiville, 2000). L'énoncé du projet constitutionnel de Charte de l'environnement en France s'y rattache également ${ }^{8}$.

L'approche stratégique du $P P$ prend le contre-pied de cette approche tactique sous deux aspects : son horizon temporel et son rapport au développement des connaissances. Abandonnant l'idée qu'une clarification scientifique suffisante, sans parler de certitudes scientifiques, pourra être obtenue à temps pour orienter les choix collectifs, ses promoteurs demandent l'adoption d'une démarche de planification d'une politique de prévention organisée à partir de la fixation d'objectifs de moyen et long termes. Cette visée de

\footnotetext{
${ }^{8}$ Ce texte énonce que, par application du $P P$, les autorités publiques doivent veiller à l'adoption de mesures provisoires...
} 
planification à long terme s'inscrit en fait dans le prolongement de l'inspiration initiale du Vorsorgeprinzip en Allemagne, ancêtre du PP (Boehmer-Christiansen, 1994). Ici les objectifs fixés sortent manifestement $\mathrm{du}$ registre $\mathrm{du}$ "provisoire » et sont rendus peu sensibles aux évolutions scientifiques qui, sauf circonstances exceptionnelles, n'auront d'effets que sur le choix des moyens et sur le rythme donné à la politique. Le calendrier daté des actions de précaution est alors déterminé principalement par la disponibilité de moyens technologiques, par les coûts en jeu et par l'acceptation sociale des risques. Le centre de gravité de la démarche se déplace de l'attention accordée au progrès de la connaissance du risque vers l'attention donnée à l'évolution des moyens technologiques et économiques disponibles pour les prévenir (invention de substituts, de nouveaux procédés de traitement, etc.). Il en résulte également une formulation différente du problème de la proportionnalité, puisque le $P P^{\text {stra }}$ se coule alors dans une logique coûts-efficacité en ne mettant en jeu dans la pesée que le choix des moyens et de la trajectoire, pas la visée ultime qui est posée d'emblée comme exogène, sauf découvertes scientifiques ou technologiques majeures ${ }^{9}$.

Comme la précédente, l'approche $P P^{\text {stra }}$ connaît plusieurs modalités selon la conception de base retenue. Pour $P P_{\text {eradic }}^{\text {stra }}$, il s'agit d'éradiquer progressivement les différentes sources de risques pour l'environnement et la santé publique, afin de parvenir à terme à une quasi-absence de risques environnementaux d'origine anthropique. Tout à fait minoritaire dans l'ordre juridique actuel, cette approche du $P P$ a néanmoins marqué de son empreinte certains textes ayant valeur d'engagement politique. Ainsi, la déclaration ministérielle adoptée lors de la réunion d'Esbjerg en 1995 en vue de protéger la mer du Nord affirme :

«Les Ministres s'accordent pour prendre comme but l'établissement d'un écosystème de la mer du Nord qui soit sain, viable et soutenable. Le principe directeur pour réaliser cet objectif est le principe de précaution. Cela implique de prévenir la pollution de la mer du Nord par une réduction continue des rejets, émissions et pertes de substances toxiques. $\grave{A}$ cette fin, il faut aller vers l'objectif de leur cessation complète en une génération (25 ans) de façon à ramener les concentrations dans l'environnement à un niveau proche des valeurs naturelles pour les substances naturellement présentes dans le milieu, et du niveau zéro pour les substances de synthèse produites par l'homme».

Le $P P$ n'est manifestement pas conçu ici comme un moyen de gérer l'attente de meilleures connaissances scientifiques...

Il existe un recours plus fréquent au $P P_{\text {inst }}^{\text {stra }}$. Il ne s'agit pas ici d'éradiquer les sources du risque suspecté mais de le contenir à terme à un niveau jugé acceptable. C'est ainsi que la Convention cadre sur le climat adoptée à l'occasion du Sommet de Rio de Janeiro en 1992 pose l'objectif d'éviter une interaction dangereuse avec les mécanismes qui régissent le climat de la planète (Godard et Henry, 1998; Guesnerie, 2003). Dans la mesure où les connaissances scientifiques disponibles ne permettent pas de déterminer de façon précise et justifiée un seuil de concentration atmosphérique qui serait un seuil de dangerosité à ne pas dépasser et, partant, une contrainte de volume sur le total des émissions admissibles d'ici 2100 , différentes autorités gouvernementales ont commencé à poser des objectifs de long

\footnotetext{
${ }^{9}$ Par exemple si l'on inventait une technique peu coûteuse de séparation et de confinement du carbone lors de la combustion d'hydrocarbures, les objectifs à long terme de réduction des émissions de gaz à effet de serre seraient bouleversés.
} 
terme. En novembre 2002, relayant le Premier ministre britannique, le Premier ministre français indiquait ainsi un objectif de division par quatre des émissions du pays d'ici 2050 par rapport au scénario de référence. De façon moins ambitieuse et moins lointaine, le protocole de Kyoto, qui n'est pas encore ratifié par assez de pays en juin 2003 pour être en vigueur, fixe aux pays de l'Union européenne un objectif de réduction des émissions de 8\% en 2008-2012 par rapport à 1990 pour six gaz à effet de serre. Si ce texte entre en vigueur, l'objectif collectif fixé à l'horizon d'une décennie ne sera pas affecté par l'évolution des connaissances.

Ces différentes conceptions du $P P$ sont récapitulées dans le Tableau 1.

\begin{tabular}{|c|c|c|}
\hline & $P P$ tactique $P P^{t a c}$ & $P P$ Stratégique $P P^{\text {stra }}$ \\
\hline$P P$ proportionné $P P_{\text {inst }}$ & $\begin{array}{c}\text { Mesures provisoires } \\
\text { proportionnées à la plausibilité } \\
\text { scientifique } P P_{\text {inst }}^{\text {tac }}\end{array}$ & $\begin{array}{l}\text { Objectifs à moyen et long termes } \\
\text { quant au niveau de risque } \\
\text { acceptable }- \text { Choix des moyens } \\
\text { sensible à l'évolution des } \\
\text { technologies } P P_{\text {inst }} \text { stra }\end{array}$ \\
\hline $\begin{array}{l}\text { Approche } \\
\text { précautionneuse } P P_{\text {prec }}\end{array}$ & $\begin{array}{l}\text { Surenchère dans la prévention } \\
\qquad P P_{\text {prec }}\end{array}$ & $\begin{array}{l}\text { Objectifs plus sévères à moyen et } \\
\text { long terme et éviction de certaines } \\
\text { substances ou activités } P P_{\text {prec }} \text { stra }\end{array}$ \\
\hline $\begin{array}{l}\text { Approche éradicatrice } \\
\text { PP eradic }\end{array}$ & $\begin{array}{c}\text { Recherche immédiate du risque } \\
\text { « absolument minimum » } \\
P P_{\text {eradic }}^{\text {tac }}\end{array}$ & $\begin{array}{c}\text { On vise le quasi risque zéro à long } \\
\text { terme } P P_{\text {eradic }} \text { tac }\end{array}$ \\
\hline $\begin{array}{l}\text { Règle d'abstention } \\
P P_{a b s}\end{array}$ & Moratoire $P P_{a b s}{ }^{t a c}$ & $\begin{array}{l}\text { Interdiction définitive d'activités } \\
\text { dont l'innocuité à long terme n'est } \\
\text { pas prouvée } P P_{a b s} \text { stra }\end{array}$ \\
\hline
\end{tabular}

Tableau 1 : les différentes conceptions du principe de précaution

La règle d'abstention $P P_{a b s}$, qui demande à ce que ne soient autorisés que les produits, techniques et activités dont l'innocuité à long terme aurait été prouvée, est la limite de l'approche précautionneuse $P P_{\text {prec }}$. Correspond-elle à une conception défendable?

\section{EST-IL RAISONNABLE DE DEMANDER LA PREUVE DE L'INNOCUITE ?}

Pour certains groupes associatifs de défense de l'environnement ou des victimes de catastrophes sanitaires, le $P P$ dont ils demandent l'application ou dont l'absence de mise en oeuvre passée leur paraît coupable sinon délictueuse ferait de la preuve de l'absence de risques ou de la preuve de l'innocuité une condition sine qua non de la légitimité d'une activité, d'une technique ou d'un produit et, par voie de conséquence, des autorisations publiques données à ces derniers. Il s'agit là du $P P_{a b s}$.

\section{Une source philosophique : le principe responsabilité de Hans Jonas}

L'idée directrice du $P P_{a b s}$ est souvent défendue à partir des idées proposées par le philosophe allemand Hans Jonas dans son livre sur le principe responsabilité [1990]. Visant les risques apocalyptiques mettant en jeu la survie de l'espèce humaine, soit à travers la destruction des bases de la vie sur terre, soit à travers une altération essentielle de ce qui fait l'humanité de 
l'homme, Jonas énonçait: " jamais l'existence ou l'essence de l'homme dans son intégralité ne doivent être mis en jeu dans les paris de l'avenir. Il en résulte automatiquement qu'ici les simples possibilités du type qui a été caractérisé (celles qui comportent un potentiel apocalyptique) sont à considérer comme des risques inacceptables qu'aucune des possibilités qui lui sont opposables ne rendent davantage acceptables" [1990, p. 62]. Jonas ajoutait: " nous devons traiter ce qui certes peut être mis en doute, tout en étant possible, à partir du moment où il s'agit d'un possible d'un certain type, comme une certitude en vue de la décision" [1990, p. 62]. Lui fait écho cette formule du Commissaire du gouvernement Legal devant le Conseil d'État dans l'affaire du sang contaminé : "En situation de risque, une hypothèse non infirmée devrait être tenue provisoirement pour valide, même si elle n'est pas formellement démontrée».

Le point critique est le suivant : de façon explicite, Jonas indiquait que sa maxime ne visait pas l'ensemble des situations à risque, mais seulement les risques potentiellement apocalyptiques. Il reconnaissait qu'on pouvait et devait raisonner autrement pour des risques qui ne l'étaient pas, même pour des risques graves engageant la vie de personnes individuelles. Godard [2002a] a montré que la norme impérative avancée par Jonas était incohérente avec d'autres éléments de la théorie de ce dernier, en particulier l'idée que le savoir prospectif sur les effets des actions humaines serait toujours en retrait par rapport à l'éventail des effets réels. Jonas en déduisait le besoin d'assister l'expertise scientifique par la mobilisation des facultés de sensibilité et d'imagination des citoyens et de leur faire partager une heuristique de la peur. S'il en est bien ainsi, il n'est jamais possible, à un stade précoce, d'avoir la certitude qu'une innovation ou un événement ne conduira jamais à une issue apocalyptique ; faute de disposer d'une faculté sûre de discernement au stade des prémisses ${ }^{10}$ le refus de prendre le risque d'une issue apocalyptique devrait alors conduire au refus de tout risque. Une telle injonction ne peut pas appartenir à notre monde ! C'est pourtant l'application de cette norme, que Jonas avait formulée pour des risques potentiellement apocalyptiques, que certains acteurs veulent obtenir pour tous les risques touchant à l'environnement et à la santé humaine. Dans sa formulation explicite sous la forme du $P P_{a b s}$ il ne s'agit plus seulement d'éviter l'apocalypse, mais d'éviter tout risque de dommage! Plus cohérente avec l'idée d'une impossibilité de faire un tri précoce entre actions, elle n'en débouche pas moins sur une impossibilité pratique comme norme générale.

\section{Des objections contre la demande de preuve de l'innocuité}

La demande de preuve de l'innocuité ou de l'absence de risques soulève de nombreuses objections, familières aux économistes :

(a) il est impossible de faire du dommage zéro une norme générale compte tenu de la rareté des moyens disponibles pour faire face à l'ensemble des risques potentiels ;

(b) il est logiquement impossible d'apporter la preuve préalable de l'innocuité d'un produit, d'une substance ou d'une technique, lorsque les connaissances scientifiques en jeu ne sont pas stabilisées ; cette impossibilité est d'abord le reflet des incertitudes existantes et des progrès incessants apportés dans le temps par la recherche scientifique; elle tient aussi au fait qu'une partie des savoirs appliqués et des informations empiriques qui seraient requis pour apporter la preuve de l'innocuité est durablement ou définitivement hors de portée ;

\footnotetext{
${ }^{10} \mathrm{Il}$ y a là un écho moderne à la parabole évangélique du bon grain et de l'ivraie.
} 
c'est pourquoi contrairement à certaines affirmations (O'Riordan \& Jordan, 1995) la véritable caractéristique du principe de précaution ne consiste pas à inverser la charge de la preuve mais à amener le processus de prise de décision à prendre ses distances vis-à-vis du concept de preuve scientifique, que cette dernière soit à charge - il faudrait prouver l'innocuité - ou à décharge - il faudrait prouver le dommage - ;

(c) les scénarios du pire qui devraient logiquement absorber la décision selon la règle d'abstention (traiter une possibilité comme une certitude et ne rien faire qui puisse conduire à cette possibilité de dommage nécessitent que le scénario du pire soit sans dommage) sont en fait des construits contingents dépendant de l'imagination des scénaristes, comme le reconnaissait Jonas qui en appelait à la mobilisation de «la lucidité de l'imagination et de la sensibilité du sentir» pour pallier les déficiences de la science des prédictions hypothétiques [1990, p. 306]; de plus, se met en place comme chez Jonas une logique de "nivellement par le pire » qui enlève à ces scénarios toute capacité de hiérarchisation entre les actions : si chaque action peut mener à l'apocalypse, et que la simple possibilité de cette issue doit être tenue pour une certitude au moment de choisir une action, la référence à l'apocalypse en vient à perdre toute pertinence pratique, puisqu'elle ne permet pas de choisir.

Ainsi prise à la lettre, la règle d'abstention n'est pas défendable. Elle tend cependant à subsister sous différentes formes atténuées qui la rendent apparemment moins déraisonnable aux yeux de ses promoteurs :

- le risque zéro n'existant pas, comme chacun va le répétant, il s'agirait quand même de l'approcher, c'est-à-dire de lui donner le statut d'une asymptote ; le $P P_{a b s}$ serait rabattu sur le $P P_{\text {eradic }}$ en exigeant l'accumulation de mesures précautionneuses susceptibles de réduire le risque autant que faire se peut; il faudrait prendre « un maximum de précautions $\gg(1)$.

- la preuve de l'absence de risques n'aurait à être apportée que dans les limites de l'état actuel des connaissances scientifiques (2);

- l'inversion de la charge de la preuve ne devrait pas être comprise comme imposant au promoteur d'un projet ou d'un produit d'apporter la preuve de l'absence de risque, mais seulement la charge de réaliser les études scientifiques nécessaires et de constituer le dossier d'expertise permettant aux autorités d'évaluer le caractère acceptable du projet ou du produit; la charge de la preuve deviendrait la charge de l'instruction du risque (3).

La thèse défendue ici est qu'il ne suffit pas d'atténuer les exigences constitutives du $P P_{a b s}$ selon les trois voies indiquées pour y voir une expression correcte et défendable du $P P$.

\section{Le PP ne consiste pas à prendre un maximum de précautions}

La discussion du premier point pourra être brève à ce stade : définir la précaution par le « maximum de précautions » ou même seulement par « davantage de prévention », constitue une entrée tout simplement différente des définitions données dans les textes juridicopolitiques qui caractérisent l'attitude à adopter face à des risques non avérés - « l'absence de certitudes ne doit pas retarder ... »-. Or ces textes ont été validés par des instances politiques (chefs d'État et de gouvernement européens) et politico-administratives (Commission européenne) aussi bien que par des textes juridiques normatifs (loi Barnier 95-101). Peut-être 
convient-il d'être très prudent et restrictif face à telle ou telle innovation, mais le $P P$ n'a pas été inventé pour sous-tendre un tel jugement. Quel que soit le niveau de sévérité des mesures de prévention jugé approprié, le $P P_{\text {inst }}$ demande essentiellement qu'on ne retarde pas leur mise en oeuvre en prétextant l'absence de certitudes scientifiques sur les risques suspectés. Il reste à savoir ce que sont les mesures proportionnées à adopter de façon précoce. C'est l'objet de la section 3 que d'en discuter.

\section{Le non sens d'une exigence de preuve concernant un risque potentiel}

Seules des connaissances stabilisées donnant leur consistance à des risques avérés sont compatibles avec l'idée de preuve. Exiger la preuve de l'absence de risque, en l'état des connaissances scientifiques, suppose donc de restreindre aux seules connaissances avérées le domaine des connaissances et informations scientifiques qui peut être pris en compte pour déterminer les mesures de précaution. Cependant, s'en tenir aux seuls tests qui s'appuient sur un domaine de connaissances ainsi restreint n'est pas de nature à fonder une démarche de précaution, puisque cela revient à ignorer toutes les informations scientifiques qui se situent dans l'intervalle entre l'ignorance et les connaissances avérées. Or le $P P$ vise précisément à organiser l'action pour les situations cognitives inscrites dans cet intervalle. Ce serait un résultat assez étrange que de réduire ainsi la précaution à l'approche classique de la prévention et, en quelque sorte, de lui couper l'herbe sous le pied! La solution de repli avancée peut donc être doublement attaquée: parce que la référence au dommage zéro contrevient à l'exigence de proportionnalité dans un contexte de moyens rares ; parce qu'elle restreint les connaissances mobilisables aux seules connaissances stabilisées, en porte-à-faux avec les circonstances d'application du $P P$.

\section{Il n'y a pas lieu d'instaurer une règle générale d'inversion de la charge d'instruction scientifique du risque potentiel}

Transformer l'inversion de la charge de la preuve en inversion de la charge de l'instruction du risque ne peut pas être défendue comme norme générale et, par voie de conséquence, comme une caractéristique intrinsèque du $P P$. C'est ainsi que la Commission européenne ${ }^{11}$ s'est refusée à prendre une position systématique d'imputation des obligations d'instruction scientifique des risques, celles-ci devant être appréciées, selon elle, en fonction des régimes d'approbation préexistants. Là où existe un régime d'autorisation préalable à la mise sur le marché (cas des médicaments, des produits chimiques et des additifs alimentaires), il appartient déjà aux entreprises d'apporter la preuve que leurs produits sont sûrs au regard des tests existants et des connaissances validées qui les sous-tendent. Sous l'égide du $P P$, il leur appartiendrait en plus d'évaluer les risques potentiels qu'ils auraient pu identifier à partir de travaux scientifiques et de présenter une argumentation sur leur acceptabilité. En revanche, lorsque des procédures d'autorisation préalable ne sont pas de mise, ou que les produits suspectés sont déjà sur le marché, pour avoir été antérieurement autorisés, c'est aux consommateurs, aux ONG ou aux autorités publiques d'apporter les éléments scientifiques nécessaires à l'analyse des risques potentiels qu'ils suspectent. Ces agents devraient alors montrer que ces données scientifiques suggèrent une possibilité plausible que les niveaux de

\footnotetext{
${ }^{11}$ Voir la Communication de la Commission de février 2000.
} 
risques en jeu ne soient pas acceptables, ce qui devrait entraîner l'adoption de mesures spécifiques de précaution. C'est donc au cas par cas que, d'après la Commission, les questions d'imputation de la charge de l'instruction scientifique devraient être réglées. Cela signifie bien que le $P P$ ne se caractérise ni par l'une ni par l'autre des options d'imputation.

En fonction de quels arguments de fond devrait-on procéder? Il y aurait lieu de prendre en compte plusieurs variables :

\section{(a) les capacités respectives des acteurs à rassembler les informations nécessaires.}

Selon une des hypothèses standard de beaucoup de modèles économiques, mais aussi de raisonnements juridiques, les agents créateurs de risques sont ceux qui disposent des meilleures informations sur les risques qu'ils engendrent. C'est donc à eux qu'il faudrait imputer la charge d'instruire le dossier des risques. Cette solution soulève toutefois un problème classique de principal-agent et fait planer une menace de capture informationnelle du principal par les agents à travers l'expertise scientifique. Les pouvoirs publics peuvent bien instaurer une obligation d'information et d'alerte, comme on en trouve l'énoncé dans le règlement européen sur la sécurité alimentaire ${ }^{12}$, cela ne règle pas le problème de l'incitation à engager des démarches scientifiques permettant d'identifier, puis de mieux connaître les risques possibles. Par ailleurs, s'il est vrai que ces agents sont souvent les mieux placés pour connaître la source de risques identifiés et avérés, ils ne sont pas nécessairement les mieux placés pour connaître les risques eux-mêmes, s'agissant de risques potentiels et, qui plus est, de risques collectifs pour l'environnement et la santé publique. Par exemple il n'y a guère de raisons de penser qu'un cimentier, gros émetteur de gaz à effet de serre, soit le mieux placé pour connaître la menace effective que l'accumulation de ces gaz fait peser sur le climat de la planète et sur tel ou tel pays (Bangladesh...), ou que les fabricants de farines animales soient les mieux placés pour identifier et évaluer les risques de développement de l'épidémie de l'ESB et de sa transmission à l'espèce humaine. Dès lors que les créateurs de risques ne seraient pas les mieux placés pour connaître les risques collectifs qu'engendre leur activité, ou qu'ils ne seraient pas incités par les règles en vigueur à développer les connaissances de ces risques, ou qu'ils seraient mis en position de pouvoir user stratégiquement du rôle qui leur serait confié au stade de l'expertise, il ne serait pas opportun de leur attribuer, à eux seuls, la charge de l'instruction des risques, bien qu'il demeure important qu'ils soient soumis à une obligation d'alerte.

(b) les exigences de la transparence de l'information et les conditions nécessaires à l'établissement d'une confiance dans l'expertise rendue.

Même si les agents créateurs du risque sont crédités de la meilleure capacité ou de la meilleure information pour identifier les risques de façon précoce, et qu'ils assument honnêtement les tâches ainsi confiées, ils ne sont pas les mieux placés pour assurer la crédibilité et la transparence de l'information qu'ils transmettent. L'implication des agents

\footnotetext{
${ }^{12}$ L'article 19-3 de ce règlement établit : "Tout exploitant du secteur alimentaire informe immédiatement les autorités compétentes lorsqu'il considère ou a des raisons de penser qu'une denrée alimentaire qu'il a mise sur le marché peut être préjudiciable à la santé humaine. Il informe les autorités compétentes des mesures qu'il prend pour prévenir les risques pour le consommateur final et n'empêche ni ne décourage personne de coopérer avec les autorités compétentes, conformément aux législations et pratiques juridiques nationales, lorsque cela peut permettre de prévenir, réduire ou éliminer un risque provoqué par une denrée alimentaire. »
} 
créateurs du risque dans l'organisation et la réalisation de l'expertise a suscité une vive défiance en France et en Europe, au point de conduire à des réformes institutionnelles qui ont attribué à des agences et à des comités "indépendants " ${ }^{13}$ le soin de réaliser lesdites expertises. C'est ainsi que le règlement européen 178/2002 créant l'Autorité européenne de sécurité des aliments [2002] stipule dans son article 22.7 : "L'Autorité exécute sa mission dans des conditions lui permettant d'assurer un rôle de référence par l'indépendance et la qualité scientifique et technique des avis qu'elle rend et des informations qu'elle diffuse, par la transparence de ses procédures et modes de fonctionnement, et par sa diligence à s'acquitter des tâches qui lui sont confiées ». Il lui incombe notamment "d'exprimer de manière autonome ses propres conclusions et orientations sur les questions qui relèvent de sa mission » (article 23-k). Dans certaines circonstances dégradées du point de vue des relations sociales, la confiance dans l'expertise ne peut être rétablie qu'en associant, étroitement et en amont, au recueil des données et à la définition du protocole d'expertise des membres ou représentants des groupes de population se sentant concernés par les risques en jeu. Il convient alors de distinguer le partenariat dans la conduite de l'expertise de l'imputation de la charge financière correspondante: l'expertise du risque serait rendue dans un cadre institutionnel à bonne distance des agents créateurs de risques, tandis que le financement de ces travaux pourrait être imputé aux entreprises qui tirent avantage des activités génératrices des risques suspectés.

(c) la répartition de cô̂ts irrécupérables associés au développement d'une technologie ou d'un produit entre les différents agents concernés.

Il existe une troisième variable, plus classiquement économique, à prendre en compte pour les choix d'imputation: le poids des coûts irrécupérables engagés par les agents créateurs de risques potentiels. De ce point de vue deux situations se distinguent nettement : celle d'un produit déjà autorisé dans le passé et très largement présent sur le marché ; celle d'un produit ou d'une substance nouvelle qui doit s'insérer dans une procédure d'autorisation préalable à la mise sur le marché. Dans le premier cas l'agent a, par hypothèse, déjà supporté un ensemble de coûts fixes faiblement récupérables à court terme, comme les coûts de $\mathrm{R} \& \mathrm{D}$, mais aussi les coûts d'investissement dans une production et une distribution qui ont été autorisées. Dans le second cas, il n'a encore engagé que les coûts de R \& D, sans validation de l'acceptabilité sociale de l'innovation proposée.

Le fait qu'une autorisation ait été donnée dans le passé n'empêche pas que cette autorisation puisse être remise en question au vu d'informations nouvelles sur des risques, mais on ne peut alors pas ignorer que des investissements ont le plus souvent été consentis sur la base de cette autorisation. Des pertes irrécupérables seraient imposés à l'agent si l'autorisation venait à être suspendue, voire annulée, dans le même temps où la charge de l'instruction des nouveaux risques suspectés lui serait attribuée.

\footnotetext{
${ }^{13}$ Indépendance et transparence sont des leitmotivs en matière d'expertise, mais le sens à donner à ces demandes n'est pas toujours clair. On peut en donner plusieurs interprétations, qui ne sont pas également défendables, par exemple lorsque le qualificatif « indépendant » est seulement attribué aux opposants à une expertise menée dans un cadre institutionnel, ou lorsque l'indépendance est attendue de l'absence d'attaches des personnes exerçant un rôle d'expert, ce qui est proprement impossible à satisfaire ; ce leurre ne peut être surmonté qu'en déplaçant la question de l'indépendance, de la qualité des experts individuels vers la qualité du processus d'expertise collective; il y a aussi de bonnes raisons de ne pas viser une transparence et une indépendance maximales (Godard, 2001, 2002b).
} 
Dans ces circonstances, on peut juger tout à la fois inéquitable et peu avisé d'imputer aux entreprises déjà investies sur un marché la réalisation de l'instruction scientifique de risques potentiels qui pourrait conduire les autorités à interdire ce qu'elles avaient précédemment autorisé ${ }^{14}$. Bernanke [1983] et Dixit [1989] avaient pointé l'effet de l'engagement de coûts irrécupérables sur la manière de traiter l'information sur les « bonnes » et les « mauvaises » nouvelles. Avant de s'engager, l'investisseur est enclin à donner plus d'importance aux « mauvaises » perspectives qu'aux «bonnes », l'inverse se produisant une fois l'investissement irrécupérable réalisé. Cela expliquait à leurs yeux les phénomènes d'hystérésis dans les réactions des investisseurs face à un avenir incertain. Dans les deux cas, le changement de poids donné à l'information se traduit par une inertie cognitive et pratique et, notamment, par des délais avant que l'investisseur ne se décide à changer d'état. L'agent porteur du projet est alors a priori beaucoup plus enclin à considérer et étudier les risques associés à son innovation dans la phase qui précède l'autorisation qu'après s'être investi dans sa production et sa mise sur le marché. On sera donc fondé à faire une imputation différente de la charge de l'instruction des risques potentiels selon que la situation est abordée avant ou après l'engagement de tels coûts par les acteurs concernés.

En conséquence, il paraît légitime, au niveau des principes, que des règles différentes s'appliquent aux produits et technologies nouveaux et à ceux dont l'usage est déjà largement répandu. Il y a là une limite à mettre aux principes de comparaison et de cohérence mis en avant par la doctrine institutionnelle du $P P$, en dépit de l'apparente irrationalité provoquée par un traitement différencié ${ }^{15}$.

Des trois atténuations considérées pour sauver le $P P_{a b s}$, seule la première n'a pas été récusée en tant que telle, puisque j'ai seulement souligné qu'il s'agissait d'un concept différent de $P P_{\text {inst }}$. Il est donc temps de la considérer au fond.

\section{COMMENT COMPRENDRE L'EXIGENCE DE PROPORTIONNALITE ?}

L'étude de la relation entre la précocité de la prise en compte des risques et la sévérité des mesures de précaution passe par l'élucidation de la manière de comprendre l'exigence de proportionnalité. Cette exigence est inscrite dans la définition légale du principe de précaution en France (loi Barnier de 1995, puis Code rural et Code de l'environnement), mais aussi dans les textes européens, et en particulier dans le règlement 178/2002 sur la sécurité alimentaire ${ }^{16}$. Doit-on comprendre que précocité et proportionnalité sont deux idées absolument indépendantes ou bien existe-t-il de bonnes raisons pour que le timing de la prise en compte

\footnotetext{
${ }^{14}$ Cela n'empêche pas ces agents de réaliser librement de telles études et analyses de risques, par exemple dans le but de disposer d'un savoir suffisant pour suivre de façon vigilante le processus d'instruction scientifique qui, par hypothèse, serait mené sous la responsabilité d'autres agents, ou de prendre de façon volontaire des mesures ciblées sur les risques suspectés.

${ }^{15}$ Ainsi entend-on souvent la question : pourquoi imposer aux OGM des tests et des procédures d'acceptation qui mettraient à la peine de nombreux produits et techniques agricoles largement utilisés ?

${ }^{16}$ L'article 7, dédié au $P P$, de ce règlement stipule au paragraphe $2:$ "les mesures adoptées en application du paragraphe 1 sont proportionnées et n'imposent pas plus de restrictions au commerce qu'il n'est nécessaire pour obtenir le niveau élevé de protection de la santé choisi par la Communauté, en tenant compte des possibilités techniques et économiques et des autres facteurs jugés légitimes en fonction des circonstances en question. » Rappelons qu'à la différence d'une directive, un règlement européen est d'application directe, sans avoir besoin d'une transposition dans le droit interne de chaque État membre.
} 
du risque, et en particulier sa précocité, influencent le jugement sur la nature des mesures proportionnées à adopter. En réfléchissant à ces questions, il apparait que la précocité pourrait avoir un tel effet si elle avait une influence sur la gravité perçue du risque potentiel. Mais reprenons d'abord la question de la proportionnalité.

\section{Les variables pertinentes du jugement sur la proportionnalité}

Quels sont les éléments qui doivent entrer en jeu dans l'appréciation de la proportionnalité des mesures de précaution? A s'en tenir à la doctrine européenne, il s'agit d'éléments classiques pour un raisonnement de type coûts-avantages :

(a) la gravité anticipée du risque en question, telle qu'on peut l'appréhender en l'état des connaissances; compte tenu des incertitudes existantes, le jugement prend ordinairement la forme d'une fourchette de valeurs sur différents indicateurs de dommages; par exemple pour le changement climatique, le Groupe intergouvernemental d'experts sur l'évolution du climat propose une fourchette de valeurs pour la température moyenne du globe, pour la remontée du niveau de la mer ou pour la distribution de la pluviométrie ;

(b) le niveau de sécurité que les pouvoirs publics se donnent pour objectif de garantir à la population ; tous les textes européens affirment qu'il est élevé ;

(c) le coût des mesures de précaution; cet élément du coût est légitime, même dans le contexte du $P P$, et même si les exigences de protection de la santé publique doivent se voir reconnaître un caractère prioritaire, d'après la résolution du Sommet de Nice. Les coûts à prendre en compte ne sont pas seulement les dépenses directes qu'implique telle ou telle mesure, mais s'étendent aux avantages auxquels la collectivité pourrait décider de renoncer par précaution; dans ce cadre, il peut être légitime d'interdire une substance pour des usages disposant de substituts praticables et de maintenir l'autorisation pour des usages qui en sont dépourvus.

Jusque-là, le tableau est d'un grand classicisme pour un économiste. On n'avait guère besoin du $P P$ pour définir ces orientations sur un plan normatif général. Il en va cependant autrement sur le plan des rapports entre l'État et les agents privés en matière de gestion des risques collectifs. Jusqu'alors, en application du principe de liberté d'entreprise, il appartenait aux agents économiques individuels, et non à une procédure publique, de déterminer l'opportunité d'un développement particulier (nouveau produit, nouvelle technique), de juger si les avantages escomptés justifiaient cet engagement, et de prendre leurs risques d'entrepreneurs. La procédure publique d'analyse de risque ne considérait que le volet des risques d'un produit ou d'une technique, sans se mêler d'évaluer ses avantages. D'où un phénomène de focalisation de l'attention publique sur la question des risques. Avec le $P P, \mathrm{du}$ fait de l'existence possible et suspectée de risques collectifs identifiés pour l'environnement et la santé, l'État devrait légitimement intervenir dans l'appréciation du bien fondé des engagements privés : afin de juger si les risques méritent d'être courus ou doivent être refusés, l'instruction des actions génératrices de risques potentiels devrait s'étendre aux avantages économiques et sociaux attendus de ces actions et ne pas se cantonner aux seuls risques de dommages pour des tiers. Cela veut dire que les innovations qui peuvent créer de nouveaux risques collectifs devraient être passées au crible de leur utilité potentielle afin de rendre possible l'appréciation proportionnée des risques en question. Je reviendrai plus loin sur le 
caractère essentiel de cet élargissement du domaine soumis à l'évaluation publique, en dépit des très grandes réticences manifestées par les milieux industriels sur ce point.

Le quatrième élément à faire intervenir est plus spécifique de la démarche intellectuelle du PP. Il s'agit de la plausibilité ou de la consistance scientifique des hypothèses de risques. Cet élément s'impose du fait du domaine de pertinence particulier du $P P$ qui est borné à ses deux extrémités en excluant tout à la fois l'état d'ignorance et le stade du risque avéré. Que l'ignorance soit simple - on ne sait pas, on ne doute pas - ou réflexive on ne sait pas mais on se demande néanmoins si le non-savoir ne cache pas un risque inconnu -, il ne lui est associé aucune identification d'un risque potentiel précis ${ }^{17}$; elle ne peut donc déboucher sur une aucune mesure proportionnée de précaution. Quant au risque avéré à la fois dans son existence et sa probabilité d'occurrence, il relève du champ de la prévention et constitue le domaine de choix de l'assurance. Le $P P$ ne trouve sens que pour des risques potentiels identifiés mais non avérés. La dimension de plausibilité ou de consistance scientifique des hypothèses de risque est alors un descripteur essentiel des contextes relevant du $P P$. Elle ne peut pas se rabattre, par définition, sur le concept de probabilité.

Entre l'état d'ignorance et le risque avéré, il existe un ensemble de risques identifiés comme possibles qui en sont à des stades variés de développement des connaissances, depuis la simple conjecture scientifique : «Tiens si ... " jusqu'à « Il y a convergence des modèles théoriques et des indices empiriques pour rendre très plausible l'existence du risque que...» (voir la section 1). A moins de considérer comme certaine toute hypothèse de risque non invalidée, la graduation de la consistance scientifique du risque importe pour définir les mesures de précaution appropriées. Mais dans quelle direction?

Rappelons que pour la doctrine institutionnelle, telle qu'on la trouve énoncée dans le rapport Kourilsky-Viney [2000], il faut que les hypothèses de risque aient atteint un niveau suffisant de plausibilité scientifique pour autoriser des mesures restrictives des libertés. Dans cette lignée, toutes choses étant égales par ailleurs sur les autres dimensions pertinentes et notamment la gravité et les coûts, la sévérité des mesures de précaution devrait être d'autant plus grande que les hypothèses sur l'existence du risque ont une plausibilité élevée. On ne voit pas en effet comment il pourrait être justifié, en raison, d'adopter des mesures d'autant plus sévères que les hypothèses de risque seraient moins scientifiquement consistantes, si la dimension de la plausibilité scientifique est indépendante des autres. En particulier on ne voit pas ce qui pourrait justifier l'idée directrice du $P P_{\text {prec }}$ ou de ses dérivés plus extrêmes, selon laquelle le $P P$ imposerait, pour des risques potentiels, plus de prévention que pour des risques avérés auxquels seraient associés le même niveau de gravité des dommages anticipés et de coûts des mesures. On en déduit l'allure générale ${ }^{18}$ de la fonction reliant alors précocité et sévérité des mesures de précaution (figure 2).

\footnotetext{
17 Quoi que certains en aient dit, le $P P$ n'a rien à faire avec ce qu'on appelle en droit le « risque de développement ». Ce concept juridique désigne la perspective que des produits ou des techniques engendrent des dommages qui seront constatés après-coup alors que le danger était scientifiquement indécelable au moment où le produit est mis en circulation ou l'équipement technique mis en service. S'agissant des produits défectueux, le législateur français en a fait un motif d'exonération de la responsabilité civile, sauf dans certains cas particuliers mettant en jeu des produits dérivés du corps humain.

${ }^{18}$ Il s'agit ici de représenter une propriété générale de la sévérité considérée comme une fonction décroissante de la précocité dans le temps scientifique et une fonction croissante de la plausibilité scientifique des hypothèses de risque. Cette représentation n'a pas la prétention de proposer une forme fonctionnelle précise et calculable.
} 


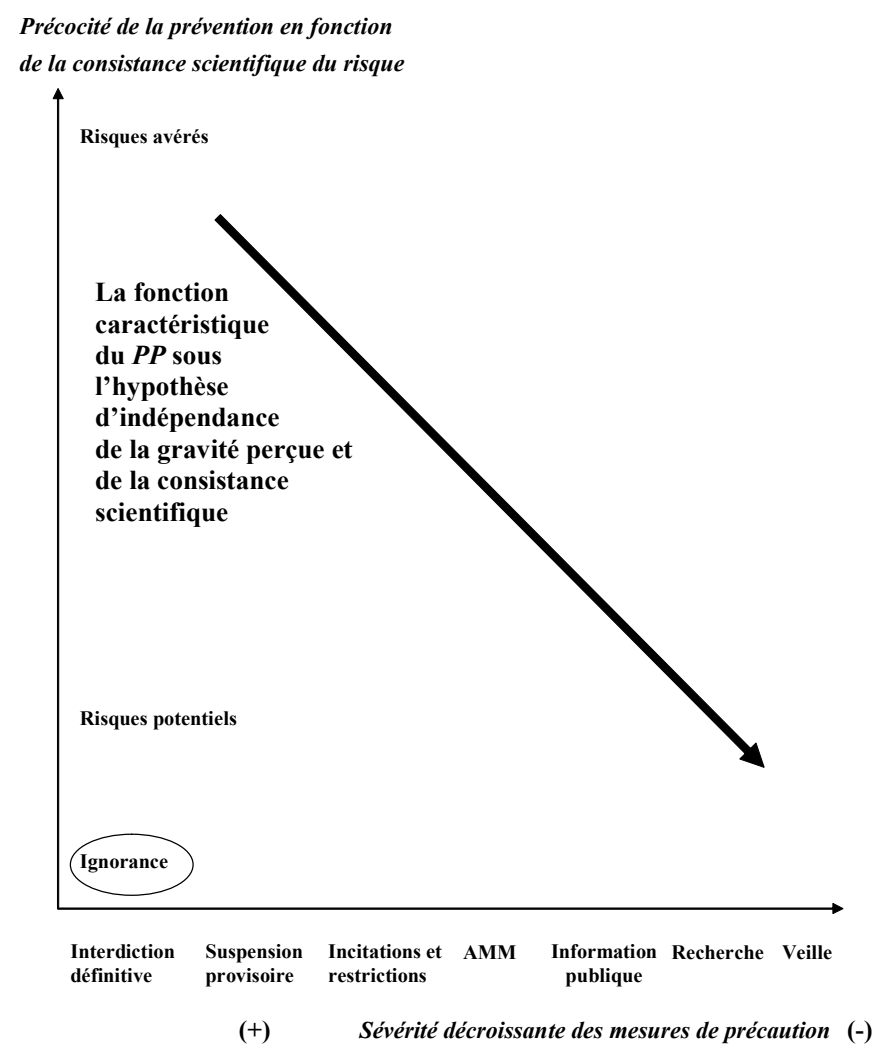

Figure 2 : la fonction caractéristique du $P P_{\text {inst }}$ sous l'hypothèse d'indépendance

Néanmoins en mettant en évidence une aversion à l'ambiguïté chez certains agents confrontés à un problème de choix dépendant à la fois d'événements aux probabilités connues et d'événements aux probabilités inconnues, le paradoxe d'Ellsberg [1961] met sur la voie, qui est celle d'un possible lien entre les différentes dimensions pertinentes de la proportionnalité. Que deviendrait le choix final des mesures de précaution si la précocité dans le temps scientifique avait une influence positive sur la gravité perçue ? On n'aurait plus affaire à des variables indépendantes : plus la prise en compte du risque serait précoce moins elle pourrait s'appuyer sur des hypothèses scientifiquement consistantes, ce qui irait dans le sens de mesures moins sévères ; mais dans le même temps la gravité perçue augmenterait, ce qui justifierait des mesures plus sévères. L'effet d'ensemble ne pourrait plus avoir la netteté postulée par le rapport Kourilsky-Viney. La conclusion précédente sur l'allure de la fonction reliant précocité et sévérité ne tiendrait plus si, pour de bonnes raisons, la précocité de la prise en compte du risque devait modifier la représentation du niveau de gravité attaché à ce risque. Plus précisément, s'il existait de bonnes raisons pour attacher au risque potentiel un niveau de gravité anticipé d'autant plus élevé que sa prise en compte serait précoce, alors il deviendrait possible de soutenir raisonnablement une fonction caractéristique d'allure inversée par rapport à la première. Cela correspondrait à l'idée directrice du $P P_{\text {prec }}$, comme cela est représenté sur la figure 3. Encore faudrait-il que, de façon systématique l'effet "aggravation du risque » l'emporte sur l'effet «dégradation de la plausibilité ». Mais ces bonnes raisons existentelles? 


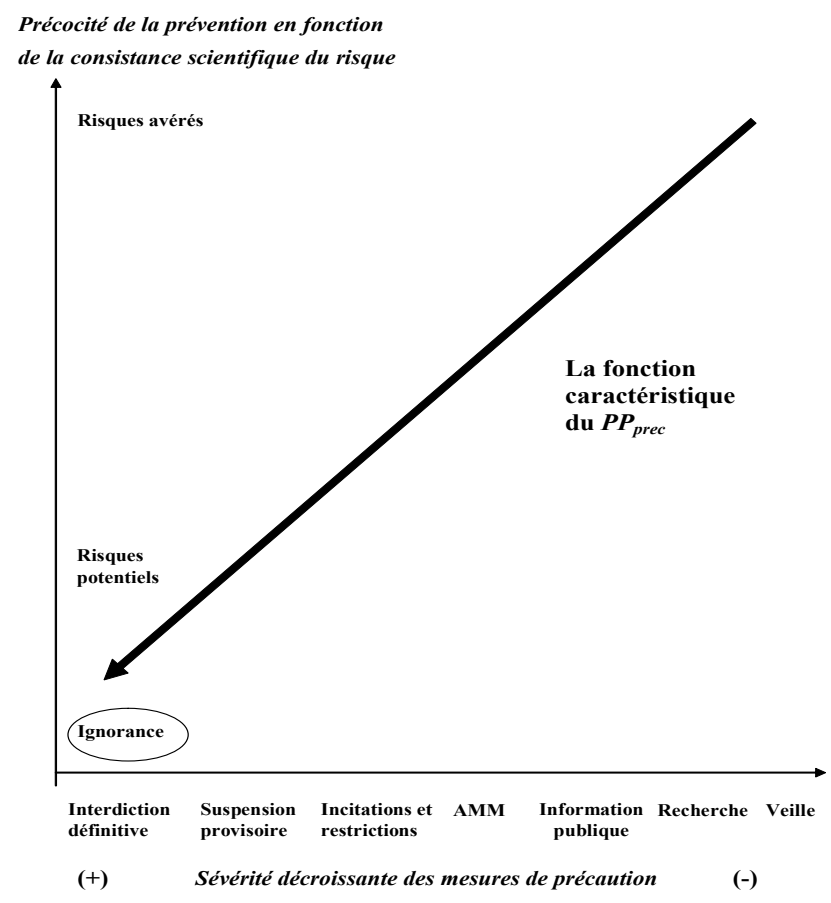

Figure 3 : la fonction caractéristique du $P P$ pour l'approche précautionneuse

La question posée attire l'attention sur la manière de structurer le problème de décision publique face aux risques potentiels. Il existe à ma connaissance deux mises en forme qui conduisent à ce résultat paradoxal d'une prévention qui aurait à être d'autant plus sévère que le risque serait appréhendé de façon précoce dans le temps scientifique. La première engendre un artefact qui fait directement dépendre la gravité perçue de l'incertitude scientifique. La seconde est ce que Gollier, Jullien, Treich [2000] ont appelé «l'effet précaution ».

\section{L'artefact d'une rétroaction amplifiante de la précocité sur la gravité perçue}

Le $P P$ vise à prendre en compte de façon précoce des risques graves et/ou irréversibles, nous disent les définitions juridiques. C'est donc au nom de la gravité intrinsèque du risque qu'il conviendrait de prendre des mesures précoces. La liaison se fait donc dans le sens gravité $\rightarrow$ précocité. Cependant en arrière-plan de cette articulation manifeste, il existe un mode de structuration du problème de choix face aux risques potentiels qui met en place une rétroaction amplifiante de la précocité sur la gravité perçue du risque. C'est le cas de la structure suivante.

Soit le mode d'appréhension du risque suivant (structure 1) : 
(a) une action potentiellement créatrice d'un risque collectif, mais aussi d'avantages économiques et sociaux tout aussi potentiels, n'est considérée dans une procédure publique d'évaluation que sous l'angle des dommages collectifs possibles; la partie 'avantages' de cette action est délibérément délaissée; aussi, au mieux, la valeur caractéristique de l'action du point de vue de la collectivité est zéro (état d'innocuité) - axiome 1-a;

(b) toute hypothèse qui n'a pas été invalidée est tenue pour valide, sans faire de distinction entre les niveaux de consistance scientifique attachés à chacune des hypothèses en présence ${ }^{19}$ - axiome $1-b$.

Sur ce fond, regardons les effets mécaniques du $P P$, appréhendé comme «prise en compte précoce de risques potentiels », par comparaison avec une situation de référence où le risque ne serait considéré qu'au moment où il serait avéré. Lorsque, sur les figures 1 à 3 , on descend le long de l'axe vertical, celui du temps scientifique de maturation des hypothèses de risque, le tableau du risque devient, par construction, de moins en moins scientifiquement consistant. Cela signifie empiriquement que le nombre d'inconnues et de questions sans réponse augmente, mais aussi que de multiples possibles surgissent ${ }^{20}$. En revanche, lorsqu'on attend que le risque soit avéré pour le prendre en compte, une possibilité au moins est confirmée tandis que d'autres sont éliminées; les possibilités à considérer sont alors en nombre plus limité ou, pour le dire autrement, les risques avérés ne sont qu'un sous-ensemble de l'ensemble des risques potentiels que l'imagination prospective peut faire surgir lorsque les travaux scientifiques ont atteint un seuil de développement suffisant pour s'éloigner de l'état d'ignorance et permettre la formulation d'hypothèses sur des risques identifiés.

Ainsi, le nombre des possibles est une fonction croissante de la précocité dans le temps scientifique. Plus on considère le risque de façon précoce, plus on multiplie les possibles à prendre en compte, cet ensemble étant constitué de toutes les hypothèses non encore infirmées. Or puisqu'on ne considère que le versant 'dommages' d'une action envisagée, et que sa valeur caractéristique est au mieux nulle, l'ouverture des possibles ne peut se faire que par l'autre bout, celui de la gravité croissante des dommages qu'elle peut entraîner. Au voisinage de l'origine, lorsqu'on sort de l'état d'ignorance quant à l'existence de risques éventuels, mais que les possibles ne sont pas bornés du côté catastrophique par une connaissance plus éprouvée qui aura pu faire le tri, toute action pourra alors être tenue pour potentiellement catastrophique, aucune hypothèse catastrophique n'ayant encore été invalidée.

C'est là qu'intervient l'axiome $1-b$ demandant de considérer comme valide toute hypothèse non infirmée. Aux stades les plus précoces de formulation des hypothèses de risque, le risque catastrophique s'impose comme possible et cette possibilité doit être tenue pour valide. Il est alors logique que face à la catastrophe annoncée, les mesures à prendre

\footnotetext{
${ }^{19}$ Cette formule fait écho à celle employée en 1993 dans l'affaire du sang contaminé par le Commissaire du gouvernement Legal devant le Conseil d'État, déjà citée : "En situation de risque, une hypothèse non infirmée devrait être tenue provisoirement pour valide, même si elle n'est pas formellement démontrée ».

${ }^{20}$ Comme l'a fait observer avec justesse un lecteur anonyme, il n'y a pas de correspondance monotone entre la graduation de la maturation scientifique des hypothèses de risque et la dynamique concrète de la recherche scientifique: à certains stades, l'avancée de la recherche peut prendre la forme d'une déstabilisation de connaissances acquises et d'un accroissement des fourchettes de valeur pour certaines variables. La découverte scientifique ne s'enferme pas dans l'idée d'affinement d'une structure d'information ; l'incertitude scientifique comprend une incertitude sur l'impact de la recherche future sur le système des connaissances.
} 
soient les plus sévères susceptibles d'empêcher sa réalisation. Amplifiant la gravité perçue des dommages, la précocité appelle donc ici des mesures d'autant plus sévères que le risque est pris en compte de façon précoce. La différence de précocité dans le temps scientifique, qui distingue la précaution de la prévention, justifierait ici qu'on caractérise le $P P$ par l'idée de surenchère précautionneuse qui sous-tend le $P P_{\text {prec }}$, comme le propose, dans des contextes analytiques différents, Treich $[1997]^{21}$ et Hermitte et Dormont $[2000]^{22}$. Le $P P_{\text {eradic }}$ serait luimême validé dans son extrémisme par une raison simple : lorsqu'ils sont considérés de la façon la plus précoce, tous les risques identifiés sont potentiellement catastrophiques... La structure $\mathrm{n}^{\circ} 1$ d'appréhension du risque rend bien compte de l'allure de la fonction caractéristique de la figure 3. Mais qu'en penser?

D'un point de vue positif, on peut avoir des doutes sur le degré d'adhésion des sujets individuels à cette structure, sinon Naples ${ }^{23}$ et San Francisco ${ }^{24}$ se seraient vidées de leurs habitants et on ne trouverait plus d'acheteur pour la viande de mouton ${ }^{25}$. Au plus fort de la crise de la vache folle, le marché des produits bovins s'est effondré (baisse des ventes de 35$40 \%$ ), mais il n'a pas disparu. En lui-même ce constat n'invalide pas la valeur normative de la maxime d'action du $P P_{\text {eradic }}$, mais elle en sape le fondement s'il était recherché du côté des attitudes les plus communes des sujets individuels vis-à-vis de risques potentiels.

Sur le plan normatif, qu'on soit adepte du conséquentialisme ou de l'équilibre réfléchi, il convient d'examiner les effets de l'application de la structure $n^{\circ} 1$ pour juger de son bienfondé. Si la prévention doit être d'autant plus sévère et couvrir un éventail de sources de risque d'autant plus large que l'incertitude scientifique est élevée, il faut alors interdire toutes les actions pour lesquelles il n'a pas été prouvé, à un stade précoce, qu'elles ne puissent pas entraîner un dommage pour la santé et pour l'environnement. On se demande, en réalité, quelles actions, quelles innovations pourraient passer au travers d'un tel crible! C'est l'argument qui avait été opposé au modèle de Jonas dans la section 2. Dans le cadre de la structure $\mathrm{n}^{\circ} 1$, la précocité rend en même temps beaucoup plus graves, voire catastrophiques, tous les risques potentiels. Il faudrait alors disposer de moyens en forte expansion pour faire face à des risques perçus comme de plus en plus graves à mesure qu'ils sont appréhendés de façon précoce. Or dans la plupart des cas, les ressources disponibles pour la prévention ne vont pas augmenter ou pas dans les mêmes proportions !

\footnotetext{
21 Treich [1997] déclare: «Notre interprétation du principe de précaution est donc la suivante: plus l'incertitude scientifique initiale est forte, plus il y a un risque de changement de perceptions, plus une attitude de prévention est nécessaire ».

${ }^{22}$ Hermitte et Dormont [2000] tirent de leur examen rétrospectif du développement de l'épidémie de l'ESB cette maxime : "Le principe de précaution implique, chaque fois que faire se peut, d'agir d'une manière d'autant plus étendue que l'incertitude est plus forte, quitte à desserrer l'étau au fur et à mesure que des résultats scientifiques le permettent. (...) Mais agir large, donc de manière coûteuse quand les indices de dérèglement sont encore très faibles, risque d'être mal compris de ceux qui auront à appliquer ces mesures et peuvent entrer en résistance ».

${ }^{23} \mathrm{Du}$ fait de la possibilité non invalidée d'un réveil catastrophique du Vésuve, risque avéré à la probabilité indéfinie.

${ }^{24} \mathrm{Du}$ fait de la possibilité non invalidée d'un tremblement de terre dévastateur, risque avéré à la probabilité mal définie à l'échelle de temps d'une vie humaine.

${ }^{25} \mathrm{Du}$ fait de la possibilité non invalidée que certaines formes de tremblante du mouton, a priori sans effet pour la santé de l'homme, soient en réalité des cas d'ESB, pathologie qui affecte l'homme sous la forme d'un nouveau variant d'une maladie, mortelle à coup sûr, la maladie de Creutzfeldt-Jakob ; l'existence de ce risque n'est ni avérée ni exclue.
} 
Sous peine d'absorber une part rapidement et fortement croissante des ressources économiques de la collectivité au détriment de la production de biens positifs, il faudra se résoudre à ce que ce soit la valeur relative des risques des uns par rapport aux autres qui importe et pas le niveau absolu de gravité perçue. En règle générale les décideurs doivent déjà arbitrer entre les actions ciblant différents risques. C'est évidemment le cas dans les situations marquées par une contrainte budgétaire donnée, comme par exemple la gestion hospitalière publique; c'est aussi vrai à l'échelle de la collectivité tout entière, toute utilisation de ressources, tout renoncement à une innovation profitable ayant un coût d'opportunité. Ce n'est donc que sous l'emprise de «l'illusion de l'isolement» d'un risque potentiel donné visà-vis de tous les autres risques potentiels qu'il est possible de donner libre cours à un double lien faisant de la gravité perçue une fonction croissante de la précocité, et de la sévérité des mesures de prévention une fonction croissante de la gravité perçue.

Pour contenir la déformation due à l'effet de loupe grossissante que produit la précocité lorsque le problème de décision est doté de la structure de décision $n^{\circ} 1$, il faut remettre en cause les deux axiomes qui composent cette dernière et adopter deux nouveaux axiomes qui, ensemble, forment la structure $\mathrm{n}^{\circ} 2$ : 1'axiome 2- $a$ pose que l'évaluation publique d'une action potentiellement créatrice de risques collectifs doit prendre en compte à la fois les dommages et les avantages possibles de l'action envisagée selon les différentes dimensions pertinentes (santé, environnement, bien-être économique, sécurité nationale et internationale, etc.) ; l'axiome $2-b$ pose que l'effet pratique à donner aux hypothèses de risques sous la forme de mesures de prévention est une fonction croissante du degré de consistance scientifique de ces hypothèses.

Dans ce nouveau cadre, le passage du risque avéré à un risque potentiel identifié, comme moment de déclenchement de la prise en compte publique d'un risque, a plusieurs effets :

- la gravité perçue des dommages potentiels continue certes à augmenter en fonction de la précocité (1);

- les avantages perçus connaissent un mouvement parallèle de grossissement : au pire, ces avantages sont nuls; l'élargissement des possibles ne pouvant se faire que par l'autre bout, la précocité de la prise en compte fait apparaître des avantages potentiels de plus en plus importants (2);

- à la précocité dans le temps scientifique correspond une dégradation de la consistance scientifique des hypothèses de risque; cette dernière diminue le poids pratique à accorder aux valeurs caractéristiques des hypothèses de risque, tant $\mathrm{du}$ côté dommages que du côté avantages (3).

L'effet d'ensemble de la précocité sur la sévérité de la prévention dépend naturellement des taux de croissance et de décroissance respectifs des facteurs « dommages », « avantages » et «consistance scientifique », si tant est qu'on puisse postuler une mesure quantitative sur ces processus. Si par hypothèse on situe le point de départ du raisonnement à l'optimum de prévention du risque avéré et que gravité des dommages et importance des avantages escomptés connaissent une croissance symétrique en fonction de la précocité, les évolutions (1) et (2) se neutralisent et l'optimum de prévention n'est pas déplacé. En revanche, l'évolution (3) réduit la sévérité des mesures qu'il est justifié de prendre et c'est ce phénomène qui détermine alors l'effet d'ensemble de la précocité sur la sévérité des mesures 
de précaution. Il est alors justifié que les mesures de prévention soient d'autant moins sévères que le risque est pris en compte de façon précoce dans le temps scientifique, ce qui correspond à l'allure de la fonction caractéristique représentée sur la figure 2 .

Pour qu'il en aille autrement, il faudrait que deux conditions soient réunies :

- l'aggravation du dommage perçu en fonction de la précocité de l'analyse se ferait à un rythme plus rapide que l'accroissement des avantages attendus ;

- la dégradation nette du bilan avantages - coûts, qui va dans le sens d'une sévérité accrue, serait plus grande, du point de vue des effets pratiques sur le choix des mesures de précaution, que la dégradation de la consistance scientifique des hypothèses de risque, qui va dans le sens d'une moindre sévérité.

Il n'y a aucune raison de voir dans cette dernière configuration l'archétype des situations de risques potentiels.

En rompant avec l'illusion de l'isolement au profit d'une approche relative de la gravité d'un ensemble de risques potentiels sous contrainte de plafonnement des ressources totales disponibles pour la prévention, et en restaurant une structure équilibrée de formulation du problème de décision, il est possible de surmonter l'aporie à laquelle conduisent les conceptions $P P_{\text {prec }}$, $P P_{\text {eradic }}$ et $P P_{a b s}$ comme normes générales, celle d'une interdiction générale de toute interaction avec l'environnement et de toute innovation comportant une incertitude. Il est possible d'admettre que la précocité de la prise en considération du risque affecte la gravité perçue de ce risque sans pour autant valider la conception de la proportionnalité véhiculée par l'idée directrice du $P P_{\text {prec }}$ et de ses dérivés.

\section{L'incertitude scientifique comme assurance de gain futur d'information}

La seconde structure du problème de décision (structure $n^{\circ} 3$ ) susceptible de soutenir une fonction croissante de la sévérité des mesures en fonction de la précocité peut être dérivée d'un modèle de Gollier, Jullien, Treich [2000], que ses auteurs présentent comme une interprétation économique du principe de précaution. Ce modèle est développé dans un cadre bayésien impliquant une révision des distributions de probabilités subjectives sur des états futurs en fonction d'événements. Les événements qui importent pour ce modèle touchent au progrès de l'information obtenu grâce au développement des connaissances scientifiques.

Ce que les auteurs appellent «l'effet-précaution » résulte de l'incidence, sur le risque perçu ex ante, de la perspective d'amélioration de l'information attribuée à l'état d'incertitude scientifique initiale : une incertitude scientifique est interprétée par les auteurs comme une promesse de meilleure connaissance future, ce qui la distingue d'un aléa pur. Les effets sur le comportement des agents en première période - nous sommes ici dans un modèle séquentiel supposant une succession de périodes auxquelles des décisions doivent être prises - sont comparés avec ce que serait le comportement des mêmes agents sans amélioration de l'information. Pour des agents dont la fonction d'utilité de Von Neumann-Morgenstern a été contrainte de façon à représenter un comportement dit « prudent» au sens de Kimball [1990], l'écart de comportements entre les situations avec et sans amélioration de l'information est attribué à « l'effet-précaution ». 
Le mécanisme est dérivé de la théorie de l'épargne. Face à l'accroissement d'un risque pour son revenu futur, un agent «prudent» augmentera son épargne courante et transférera des ressources sur la période à laquelle une amélioration de l'information est attendue. Le lien entre la situation de l'épargnant et le problème du choix face à des risques potentiels marqués par l'incertitude scientifique est noué en considérant que le savoir ex ante sur le fait que l'information va s'améliorer dans le futur accroît le risque perçu ex ante par rapport à une situation de référence ne comportant aucune perspective d'amélioration de l'information à travers le temps : du fait du progrès des connaissances attendu, l'agent anticipe que la structure d'information sur la réalisation des états futurs va s'affiner, ce qui signifie que l'état de quasi équiprobabilité qui caractérise son faible niveau d'information initiale - « tout est possible ! »- va laisser la place à une distribution plus contrastée de probabilités. L'agent s'attend donc à devoir réviser substantiellement ses croyances et ses actions, chose qu'il n'aurait pas eu à faire en l'absence d'amélioration de l'information. Du fait de l'incertitude scientifique initiale et de la promesse d'amélioration de l'information qu'elle porte, le risque ex ante est plus élevé que face à un aléa pur et un agent " prudent » épargne davantage pour se prémunir contre ce risque.

Les auteurs appliquent cette structure à un problème de risque cumulatif comme le risque climatique planétaire résultant de l'amplification anthropique de l'effet de serre : c'est la concentration atmosphérique de gaz à effet de serre qui représente la source de danger climatique, mais cette concentration dépend des flux d'émissions réalisés à travers le temps. Dans ce cadre précis, l'agent «prudent» peut faire d'une pierre deux coups : en réduisant sa consommation courante, il réduit ses émissions et donc la source du risque futur - en adoptant l'hypothèse héroïque que cet agent est en fait l'agent représentatif de la population mondiale et il transfère des ressources économiques pour son usage futur. Ainsi, loin d'être une bonne raison de ne rien changer à sa consommation courante, la situation d'incertitude scientifique constitue, pour des agents "prudents », un motif rationnel d'épargner davantage et, en l'occurrence, de faire "plus de prévention », puisque la baisse de la consommation courante réduit aussi le risque à sa source.

Comme la perspective d'une amélioration de l'information garantit à l'agent qu'il pourra faire des choix plus avisés à l'avenir, car mieux informés, elle induit également un effet-richesse qui, pour sa part devrait conduire l'agent à épargner moins qu'en l'absence d'amélioration de l'information. On retrouve ici une dualité d'effets. Puisqu'il y a deux effets de sens opposé, l'effet net demeure ambigu en toute généralité. Ce n'est que pour les fonctions d'utilité obéissant à une condition particulière : $\mathrm{P}>2 \mathrm{~A}$, c'est-à-dire lorsque la valeur de l'indicateur de prudence est supérieure à deux fois la valeur de l'indicateur d'aversion absolue au risque, que «l'effet précaution » l'emporte sur « l'effet-richesse ». Tel serait le fondement économique du principe de précaution.

Gardons les grandes lignes de ce modèle et utilisons le pour aborder le lien entre précocité et sévérité de la prévention. À mesure qu'un décideur public considère le risque à un stade plus précoce et qu'il descend le long de l'axe vertical du graphique de la figure 1, il se trouve confronté à un tableau qui est de moins en moins consistant sur le plan scientifique et qui comporte également davantage d'hypothèses possibles, chacune étant cependant moins étayée que celles qui seraient capables de demeurer en piste à un stade plus avancé du savoir. Si ce décideur est assuré que cette incertitude scientifique, qui s'accroît avec la précocité, va se résorber à l'avenir pour mettre à jour un risque avéré, il se trouve alors à peu près dans le 
cadre défini par Gollier, Jullien, Treich. Sachant que l'incertitude va se résorber à l'avenir, plus le décideur appréhende le tableau des risques de façon précoce, plus il éprouve une grande incertitude sur ce qui va lui être finalement révélé par la connaissance lorsqu'elle arrivera. S'il appartient à la classe des agents " prudents» et si les ressources épargnées lui permettent en même temps de réduire les sources de risque, il va réserver davantage de ressources pour faire face à l'imprévu dans l'avenir que dans le cas où il estime que l'incertitude demeurera inchangée.

En revanche, s'il n'a aucune assurance sur la résorption de l'incertitude scientifique ou si les actions de prévention à sa disposition ne lui permettent pas de transférer des ressources sur le futur, "l'effet précaution » se dissipe, alors même que le problème du choix d'une politique relevant du $P P$ face à des risques potentiels demeure entier.

Avec le modèle de Gollier, Jullien, Treich, ce n'est pas par le biais d'une boucle précocité-gravité qu'on trouve une explication possible à une fonction faisant de la sévérité une fonction croissante de la précocité, mais par le biais d'une incertitude scientifique interprétée comme une promesse d'amélioration future de l'information. Pour que ce mécanisme joue, il faut ici un ensemble de circonstances particulières :

- il faut d'abord une épargne qui fait d'une pierre deux coups en réduisant le risque à sa source, en supposant encore que les sources de risque sont directement proportionnelles à la dépense courante. Or dans la plupart des cas, les mesures de précaution à prendre impliquent des coûts nets immédiats (information, recherche, destruction d'équipements ou de produits, renoncement aux avantages immédiats procurés par certaines activités ou produits), sans transférer aucune ressource économique supplémentaire sur l'avenir; elles contribuent juste, on le souhaite, à améliorer la qualité du patrimoine naturel transmis aux générations à venir, ou le niveau actuel et futur de santé et de sécurité de la population;

- il faut ensuite des préférences particulières, généralement attribuées dans la théorie de l'épargne, à l'attitude psychologique de certains agents. Dans la mesure où cette attitude n'est pas partagée par l'ensemble des membres de la société ${ }^{26}$, il est difficile d'y voir le fondement du PP comme norme publique ;

- il faut enfin des perspectives crédibles d'amélioration de l'information, au point de faire de ces perspectives la clé de voûte de la stratégie de précaution. Or ce n'est pas du tout le cas général des situations pour lesquelles le $P P$ est mobilisé.

Attardons-nous sur ce dernier point. Au sein des cercles scientifiques eux-mêmes ou au sein d'ONG, nombreux sont ceux qui s'attachent à rappeler les limites cognitives importantes et durables des démarches scientifiques : la science n'a pas réponse à toutes les questions que se posent les citoyens, par exemple dans des domaines comme l'écotoxicologie et la climatologie. Ces sceptiques voient dans le $P P$ une réponse à la prise de conscience des limites structurelles de la connaissance scientifique pour fonder l'action, ce qui les conduit à vouloir donner d'autres bases aux actions de précaution. L'argument se présente à peu près de la manière suivante : les connaissances avérées arrivent toujours trop tard par rapport aux exigences de l'action et, du fait du niveau élevé de complexité des questions posées, ne sont finalement pas conclusives sur les points clés. Aussi l'insistance sur l'évaluation scientifique

\footnotetext{
${ }^{26} \mathrm{Il}$ semble même qu'elle soit empiriquement très minoritaire.
} 
des risques et sur les perspective d'amélioration de l'information résultant de programmes de recherche scientifique apparaît-elle suspecte à ces critiques : elle entretiendrait les mythes scientistes de la période historique antérieure, ou véhiculerait des stratégies visant, sous prétexte de rigueur et d'objectivité, à évacuer des demandes sociales légitimes (Santillo et al. 1998). On retrouve ici la distinction entre le $P P^{t a c}$ et le $P P^{\text {stra }}$ établie en section 1. Le modèle de Gollier, Jullien, Treich ne peut concerner que le $P P^{t a c}$, pas le $P P^{\text {stra }}$.

\section{CONCLUSION}

L'article avait une double ambition: d'abord présenter de façon ordonnée les différentes conceptions du $P P$ en présence dans le débat français et européen pour aborder des risques collectifs potentiels ; ensuite procéder à une critique des conceptions alternatives à la doctrine institutionnelle qui s'est élaborée depuis une dizaine d'années et cristallisée dans le $P P_{\text {inst }}$. Ont ainsi été mises en contraste les idées directrices sous-jacentes aux $P P_{\text {prec }}, P P_{\text {eradic }}$ et $P P_{a b s}$, puis aux $P P^{t a c}$ et $P P^{s t r a}$. C'est sur cette base qu'une démarche critique a été entreprise, d'abord en concentrant l'attention sur la conception la plus antagoniste du $P P_{\text {inst }}$ qu'est le $P P_{a b s}$ : étaitil défendable de comprendre le $P P$ comme une exigence de preuve de l'innocuité ? Une fois acquise la réponse négative donnée à cette question, l'article s'est intéressé aux formes atténuées du $P P_{a b s}$ que sont les $P P_{\text {eradic }}$ et $P P_{\text {prec }}$. Il en est ressorti que les voies proposées pour l'atténuation de l'inversion de la charge de la preuve soit n'avaient guère de sens, soit ne pouvaient être érigées en norme générale. Restait à élucider la question de la proportionnalité. Dans quel sens la faire jouer par comparaison avec le traitement standard d'un risque avéré ?

Il est apparu que l'idée de prendre des mesures d'autant plus sévères que la prise en compte des risques est précoce dans le temps scientifique dépendait de la combinaison de deux types de facteurs : (a) des phénomènes de dépendance, soit de la gravité perçue par rapport à la précocité de la prise en considération (a1), soit de l'ampleur du risque perçu ex ante par rapport à la perspective d'amélioration de l'information à l'avenir (a2); (b) des formes particulières de représentation du problème de choix d'une stratégie de précaution. Combinée au facteur (a1), une représentation tronquée du problème de décision qui se focalise sur les seuls dommages possibles et considère toutes les hypothèses de risque comme également valides (b1) met en branle un artefact de perception par lequel la précocité accroît la gravité perçue, situation qui appelle a priori des mesures plus sévères. Combinée au facteur (a2), une configuration spécifique étudiée par Gollier, Jullien, Treich [2000] (b2) conduit également à justifier, pour des agents prudents, d'autant plus de prévention que l'incertitude scientifique est susceptible de se résorber à l'avenir. Ni l'une ni l'autre de ces deux figures ne peut prétendre incarner l'idée directrice du $P P$ sur cette question de la proportionnalité. La première est impraticable comme norme générale en induisant un artefact d'aggravation générale de tous les risques potentiels dans une situation de moyens limités. La seconde combine de façon rare les attributs particuliers de préférences d'agents «prudents », qui ne sont pas représentatifs de l'ensemble de la société, une structure cognitive particulière dans laquelle les agents ont de bonnes raisons de penser que l'incertitude initiale se réduira de façon significative, et un mode d'action qui permet à la fois de mettre en réserve des ressources pour l'avenir et de réduire les sources courantes du risque. 
Le principal résultat de cet article conforte les vues développées par la doctrine du $P P_{\text {inst }}$ et reconnues par les institutions publiques européennes. Le $P P$ ne consiste pas à rechercher à atteindre asymptotiquement un état de risque potentiel zéro en promouvant un « maximum de précautions ». Son objectif ne peut pas être de tout faire pour empêcher absolument la réalisation d'un dommage potentiel, même grave et irréversible, même apocalyptique. Son idée directrice est celle d'une prévention précoce, mais proportionnée, des risques potentiels. Toutes les hypothèses de risque ne peuvent pas être considérées comme également valides. Une des tâches essentielles des comités d'experts scientifiques consiste à juger de la plausibilité ou de la consistance scientifique des hypothèses de risques en fonction d'une graduation pertinente pour l'action publique (Godard, 2002b). Selon cette graduation, il se confirme dans le cas général que moins une hypothèse est scientifiquement consistante, moins elle est plausible, moins il est justifié de prendre des mesures sévères de précaution. Ce résultat s'impose de lui-même dans une hypothèse d'indépendance de la gravité par rapport aux autres variables de proportionnalité. Il s'impose également de façon assez générale, mais pas dans tous les cas, lorsque l'appréhension de la gravité du risque dépend de la précocité de la prise en considération de ce risque sur l'axe du temps de la maturation scientifique des connaissances. C'est pourquoi il convient de résister à la tentation catastrophiste qui réduit toute hypothèse de risque à la catastrophe dont elle pourrait être porteuse, sans considération pour la plausibilité de ce scénario. Si la démarche apocalyptique inspirait vraiment la gestion publique des risques et, au-delà, le comportement de tous les agents économiques, elle absorberait l'ensemble des ressources disponibles et mettrait en crise systèmes économiques et systèmes politiques.

\section{REFERENCES}

Beck, U. [2001], La société du risque. Sur la voie d'une autre modernité. Paris, Aubier, Coll. 'Alto'.

Bernanke, B. [1983], «Irreversibility, uncertainty and cyclical investment », Quarterly Journal of Economics, 98, pp. 85-106.

Boehmer-Christiansen, S. [1994], «Chapter 2. The Precautionary Principle in Germany - Enabling Government », in T. O'Riordan and J. Cameron, Interpreting the Precautionary Principle. London, Earthscan, pp. 31-60.

Chevassus-Au-Louis, B. [2000], «L'analyse du risque alimentaire. Vers de nouvelles pratiques?», Conférence La sécurité sanitaire des aliments issus d'OGM, organisée par l'OCDE, Edimbourg (RU), 28 février- $1^{\mathrm{er}}$ mars.

Commission des Communautés Européennes [2000], Communication sur le principe de précaution, Bruxelles, $\operatorname{COM}(2000) 1,2$ février.

Conseil d'État [1998], Rapport public 1998 - Réflexions sur le droit de la santé. Paris, La Documentation française.

Dixit, A.K. [1989], «Entry and exit decisions under uncertainty », Journal of Political Economy, 97(3), pp. 620-638.

Ellsberg, D. [1961], « Risk, ambiguity and the Savage axioms », Quarterly Journal of Economics, 75, pp. 643-669.

Ewald, F., C. Gollier et N. de Sadeleer [2001], Le principe de précaution. Paris, PUF, Que-sais-je.

Godard, O. (dir.) [1997], Le principe de précaution dans la conduite des affaires humaines, Paris, Éd. de la Maison des Sciences de l'Homme et INRA-Éditions.

Godard, O. [1997], «L'ambivalence de la précaution et la transformation des rapports entre science et décision », in O. Godard (dir.), op. cit., pp. 37-83. 
Godard, O. [2001], «L'expertise économique du changement climatique planétaire », Annales des Mines - Série Responsabilité et environnement, (21), janvier, pp. 23-65.

Godard, O. [2002a], «L'impasse de l'approche apocalyptique de la précaution. De Hans Jonas à la vache folle », in Éthique Publique, 4(2), Montréal, octobre, pp. 7-23.

Godard, O. [2002b], «Expertise et gestion des risques sanitaires - Leçons tirées du maintien de l'embargo sur le bœuf britannique en 1999 », Revue française d'administration publique, 'L'administrateur et l'expert', (103), pp. 411-421.

Godard, O. et C. Henry [1998], « Les instruments des politiques internationales de l'environnement : la prévention du risque climatique et les mécanismes de permis négociables », rapport au Conseil d'analyse économique, in D. Bureau, O. Godard, C. Henry, J.-C. Hourcade, A. Lipietz, Fiscalité de l'environnement. Paris, La Documentation française, Collection des Rapports du CAE, pp. 83-174.

Godard, O., C. Henry, P. Lagadec et E. Michel-Kerjan [2002], Traité des nouveaux risques. Précaution, crise, assurance. Paris, Gallimard, Coll. 'Folio-Actuel n 100 '.

Gollier, C., B. Jullien and N. Treich [2000], «Scientific progress and irreversibility: an economic interpretation of the 'Precautionary Principle' », Journal of Public Economics, 75, pp. 229-253.

Guesnerie, R. [2003], Kyoto et l'économie de l'effet de serre. Rapport au Conseil d'analyse économique. Paris, La Documentation française.

Hermitte, M.-A. et D. Dormont [2000], « Propositions pour le principe de précaution à la lumière de l'affaire de la vache folle », in P. Kourilsky et G. Viney, op. cit., pp. 341-386.

Jonas, H. [1990], Le principe responsabilité. Une éthique pour la civilisation technologique. Paris, Cerf.

Kimball, M.S. [1990], «Precautionary savings in the small and in the large », Econometrica, 58(1), pp. 53-73.

Knight, F.H. [1921], Risk, Uncertainty and Profit, New-York, Kelley.

Kourilsky, P., et G. Viney [2000], Le principe de précaution. Rapport au Premier ministre, Paris, La Documentation française et Odile Jacob.

Lagadec, P. [1981], La civilisation du risque. Catastrophes technologiques et responsabilité sociale, Paris, Seuil, Coll. 'Science ouverte'.

Noiville, C. [2000], «Principe de précaution et Organisation mondiale du commerce. Le cas du commerce alimentaire », Journal du Droit International, 127(2), pp. 263-297.

O'Riordan, T. and J. Cameron (eds) [1994], Interpreting the Precautionary Principle. London, Earthscan.

O'Riordan, T., and A. Jordan [1995], « The precautionary principle in contemporary environmental politics », Environmental Values, 4, pp.191-212.

Parlement européen et Conseil européen [2002], «Règlement (CE) № 178/2002 du 28 janvier 2002 établissant les principes généraux et les prescriptions générales de la législation alimentaire, instituant l'Autorité européenne de sécurité des aliments et fixant des procédures relatives à la sécurité des denrées alimentaires », Journal officiel des Communautés européennes, L 31/1 du 1 février 2002.

Raffensperger, C. and J. Tickner (eds) [1999], Protecting Public Health and the Environment. Implementing the Precautionary Principle. Washington DC, Island Press.

Santillo, D., R.L. Stringer, P.A Johnston and J. Tickner [1998], « The precautionary principle: protection against failures of scientific method and risk assessment », Marine Pollution Bulletin, 36(12), pp. 939-950.

Treich, N. [1997], « Vers une théorie économique de la précaution ?», Risques, (32).

Zaccaï, E. \& J.-N. Missa (dir.) [2000], Le principe de précaution. Signification et conséquences. Bruxelles, Éditions de l'Université de Bruxelles. 